\title{
Assessment of spectral and angular characteristics of sub-GLE events using the global neutron monitor network
}

\author{
Alexander Mishev ${ }^{1, *}$, Stepan Poluianov ${ }^{1,2}$ and Ilya Usoskin ${ }^{1,2}$ \\ ${ }^{1}$ Space Climate Research Unit, University of Oulu, Oulu, Finland \\ ${ }^{2}$ Sodankylä Geophysical Observatory (Oulu Unit) University of Oulu, Oulu, Finland
}

Received 24 May 2017 / Accepted 23 September 2017

\begin{abstract}
New recently installed high-altitude polar neutron monitors (NMs) have made the worldwide NM network more sensitive to strong solar energetic particle (SEP) events, registered at ground level, namely ground-level enhancement (GLE) events. The DOMC/B and South Pole NMs in addition to marginal cut-off rigidity also possess lower atmospheric cut-off compared to the sea level. As a result, the two high-altitude polar NM stations are able to detect lower energy SEP events, which most likely would not be registered by the other (near sea level) NMs. Here, we consider several candidates for such type of events called sub-GLEs. Using the worldwide NM database (NMDB) records and an optimization procedure combined with simulation of the global NM network response, we assess the spectral and angular characteristics of sub-GLE particles. With the estimated spectral characteristics as an input, we evaluate the effective dose rate in polar and sub-polar regions at typical commercial flight altitude. Hence, we demonstrate that the global NM network is a useful tool to estimate important space weather effects, e.g., the aircrew exposure due to cosmic rays of galactic and/or solar origins.
\end{abstract}

Keywords: SEP / GLE and sub-GLE events / neutron monitor network / spectral and angular characteristics of SEPs / radiation environment

\section{Introduction}

Radiation environment in the vicinity of Earth and in the Earth's atmosphere is variable and highly dynamic (e.g. Vainio et al., 2009, and references therein). Several sources contribute to the radiation environment, including cosmic rays (CRs) of galactic and solar origin as well as radiation-belt particles, the latter specifically contribute at orbital and sub-orbital altitudes, therefore are not considered here. However, recent measurements discuss the possible contribution of radiation belt particles as well as changes in the geomagnetic field and air density to variable radiation environment in the vicinity of Earth and within the Earth's atmosphere (e.g. Lee et al., 2015; Atwell et al., 2017). In addition, some natural radio-nuclides from the Earth's crust also contribute to the atmospheric radiation, particularly in near surface region of the atmosphere (e.g. Eisenbud and Gesell, 1997; Balanov et al., 2008, and references therein).

Various populations of $\mathrm{CR}$ particles possess different characteristics such as composition and abundances, energy range and spectra, intensity, spatial distribution, time variations, occurrence rate. According to the current knowledge, galactic cosmic rays (GCRs) originate from the Galaxy, being

\footnotetext{
*Correspondence: alex_mishev@yahoo.com
}

accelerated in supernova remnants and consist mostly of protons and $\alpha$-particles with small abundance of heavier nuclei (e.g. Grieder, 2001; Gaisser and Stanev, 2010, and references therein). The GCR flux, specifically its low-energy part, is modulated in the Heliosphere, thus following the 11-year solar cycle in antiphase with solar activity and also responding to long and short-time scale solar wind variations (e.g. Dorman, 2006, and references therein). GCR particles entering into the atmosphere induce a complicated nuclear-electromagnetic-muon cascade leading to ionization of the ambient air (Usoskin \& Kovaltsov, 2006; Bazilevskaya et al., 2008; Usoskin et al., 2009).

Another important but sporadic source, which makes the radiation environment in the vicinity of Earth and within its atmosphere highly dynamical is related to eruptive solar processes, namely solar flares and coronal mass ejections (CMEs), leading to production of solar energetic particles (SEPs) (e.g. Reames, 1999; Cliver et al., 2004; Reames, 2013; Desai and Giacalone, 2016, and references therein). The energy of SEPs is usually of the order of a few tens of $\mathrm{MeV} /$ nucleon, rarely exceeding $100 \mathrm{MeV} /$ nucleon, occasionally reaching several $\mathrm{GeV} /$ nucleon. While lower energy SEPs are absorbed in the atmosphere, more energetic particles possess enough energy to initiate an atmospheric cascade similar to GCRs, which eventually reaches the ground and leads to an enhancement of count rates of ground based detectors, 
specifically neutron monitors (NMs). This special class of SEP events, called ground-level enhancements (GLEs), with the occurrence rate roughly once per year with higher probability during maximum and decline phase of the solar activity cycle (Shea and Smart, 1990; Stoker, 1995; Bazilevskaya 2005), can drastically change the Earth's radiation environment (e.g. Vainio et al., 2009, and references therein).

CR particles of both galactic and solar origin significantly affect the radiation environment in the vicinity of the Earth as well as in the Earth's atmosphere, accordingly the exposure of aircrew and passengers (e.g. Shea and Smart, 2000, and references therein). Both GCRs and SEPs are the most significant contributors to the increased radiation exposure of aircrew and airline passengers compared to the exposure at ground level, specifically over the polar regions, where the magnetospheric shielding is not as effective as at middle and equatorial latitudes. Therefore, particularly high energy SEP events, can lead to significant space weather effects. According to the common definition, space weather refers to the dynamic, variable conditions on the Sun, of the solar wind, of the Earth's magnetosphere as well as of the ionosphere and can compromise the performance and reliability of spacecraft and ground-based systems (e.g. by geomagnetically induced currents on power lines and/or pipelines) and can endanger human health (e.g. Lilensten and Bornarel, 2009). Because of the increased intensity of secondary CRs at flight altitudes, specifically during SEP events, aircrews are a subject to additional exposure, particularly during intercontinental flights over the sub-polar and polar regions. Assessment of aircrew exposure due to CRs is an important topic of space weather studies. Accordingly, a new branch in the field of radiation protection has appeared. Nowadays, aircrews are a subject of new regulations. The exposure of both cabin and cockpit crew to cosmic radiation is regarded as occupational (ICRP, 1991; EURATOM, 1996).

In order to mitigate those effects, detailed studies of SEPs, specifically their spectral and anisotropy characteristics are necessary. While the characteristics of the low energy part of GCR and SEPs can be assessed using satellite-borne measurements (e.g. Aguilar et al., 2010; Adriani et al., 2016), the higher energy part, specifically GLE particles are studied by NMs (e.g. Dorman, 2004, and references therein). In order to assess the SEP spectral and angular characteristics using measurements retrieved from the global NM network, it is necessary to model their propagation through the atmosphere and magnetosphere of the Earth (e.g. Shea and Smart, 1982; Humble et al., 1991; Cramp et al., 1997; Vashenyuk et al., 2006; Desorgher et al., 2009; Mishev and Usoskin, 2013). Moreover, a sufficient number of NM stations with nonnull response is necessary in order to perform an optimization procedure over a set of model parameters and experimental data similar to Cramp et al. (1997); Vashenyuk et al. (2006); Mishev et al. (2014).

Nowadays, after the launch of high-altitude polar NMs possessing lower atmospheric cut-off compared to the sea level stations, the worldwide NM network has become more sensitive to strong SEP events. As a result, in some cases the NM response is null and/or marginal in all stations, but at the high altitude, polar ones, e.g. South Pole and Dome C. In this case the estimation of spectral and angular characteristics of sub-GLE particles (see Sect. 2.1) is a real challenge. In this

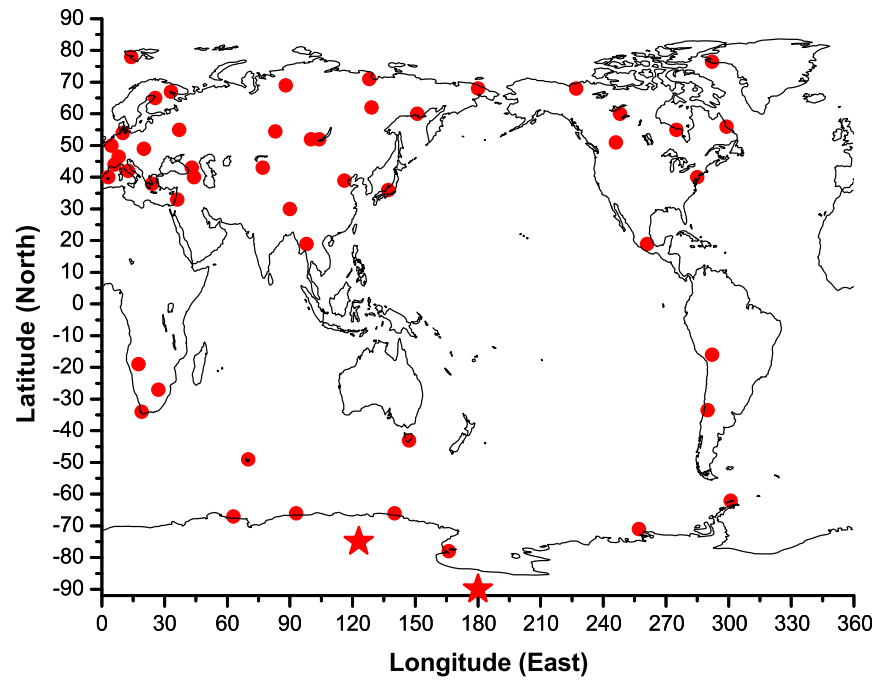

Fig. 1. Current status of the global neutron monitor network. Red circles depict the NM stations, while the red stars the two highaltitude polar NM stations at Dome C $\left(75^{\circ} \mathrm{S}, 123^{\circ} \mathrm{E}\right)$ and South Pole $\left(90^{\circ} \mathrm{S}\right)$.

work, we assess the spectral and angular characteristics of subGLE particles using the worldwide NMDB records and performing an optimization procedure combined with full simulation of the global NM response. Subsequently, we estimate the corresponding aircrew exposure using the derived spectral characteristics as an input.

\section{GLE and sub-GLE analysis using the global neutron monitor network}

NMs have been successfully used for continuous CR measurements applied for CR intensity variations and other studies (e.g. Moraal, 1976; Debrunner et al., 1988; Lockwood et al., 1990; Dorman, 2006; Kudela, 2016).

The first generation of NMs was introduced as a continuous recorder of CR intensity during the International Geophysical Year (IGY) 1957-1958 (Simpson, 1957). It usually represents a detector with 12 proportional counters, but there existed/exist IGY NMs with different number of counter tubes (Simpson et al., 1953; Simpson, 1957). The IGY NM was used worldwide as a primary detector to study $\mathrm{CR}$ variations. Subsequently, the design of NM was considerably optimized leading to an increase of the NM counting rate (Hatton and Carmichael, 1964; Hatton, 1971). This second generation of the NM design is known as NM64(for details see Carmichael H. (1968); Hatton (1971); Stoker et al. (2000) and references therein). Mini NMs were recently introduced (Krüger and Moraal, 2013; Krüger et al., 2015). They are successfully used for latitude or altitude surveys and station device(s) (Heber et al., 2015; Poluianov et al., 2015; Lara et al., 2016). Hence, the mini NMs form a part of the global NM network (Fig. 1) (Moraal et al., 2000; Mavromichalaki et al., 2011).

NMs are successfully used for the estimation of spectral and angular characteristics of GLE particles in the vicinity of Earth (e.g. Shea and Smart, 1982; Cramp et al., 1997; Bombardieri et al., 2006; Vashenyuk et al., 2006; Mishev et al., 2014). According to the generally accepted common definition, 
a GLE event is registered when there is a simultaneous statistically significant increase of the count rate of at least two differently located NM stations accompanied with a statistically significant increase of the SEP flux directly observed by a spaceborne instrument(s), accordingly a sub-GLE event is registered when there are simultaneous statistically significant increase of the count rates of at least two differently located high-altitude $\mathrm{NMS}$ with corresponding enhancement in the proton flux measured by a space-borne instrument(s), but no statistically significant enhancement in the countrates of NMs near to the sea level (Poluianov et al., 2017).

After the start of operation of $\mathrm{DOMC} / \mathrm{B} \mathrm{NMs}$, the worldwide NM network became more sensitive to the registration of SEP events. The DOMC/B and both South Pole NMs (Bieber et al., 2013), in addition to marginal cut-off rigidity possess lower atmospheric cut-offs compared to a sea level station, because of their high elevation (Tab. B1) (Carmichael et al., 1968). Hence, all high-altitude polar NMs are more sensitive to lower energy SEPs compared to sea level ones. In addition, both high-altitude polar NM stations (South Pole and Dome C) are equipped with a pair of standard and bare NMs, the latter with even better response to the low energy part of the SEP rigidity spectrum compared to a standard NM (Vashenyuk et al., 2007). As a result, the two high-altitude polar NM stations (Fig. 1) are able to detect SEP events, which most-likely would not be registered by the other (near sea level) NMs. Here, as a near sea level station we assume a NM at altitude(s) lower than of about $1000 \mathrm{~m}$ above the sea level (a.s.1.). This special subclass of events - subGLEs deserves special interest, specifically for space weather purposes.

The assessment of the spectral characteristics of sub-GLE particles is important in order to mitigate space weather effects, such as aircrew exposure. This task is a real challenge since at sub-GLE energies of about $300 \mathrm{MeV} /$ nucleon, most of the space-borne instruments saturate and/or are not efficient, while the methods based on NM records suffer of a lack of precision or are not applicable (see Sect. 2.1).

\subsection{General description of the method}

In general, the spectral and angular characteristics of SEPs on the basis of NM measurements can be derived using the relationship between NM count rate and the primary particle flux (particles arriving at the top of the atmosphere) via the NM yield function, which considers the full complexity of particle transport in the Earth's atmosphere as well as the detector response, i.e. the registration efficiency and effective detector area itself (Mishev and Usoskin, 2013; Mishev et al., 2013).

Different methods for analysis of GLEs using NM measurements have been proposed over the years (e.g. Shea and Smart, 1982; Bieber and Evenson, 1995; Humble et al., 1991; Cramp et al., 1997; Vashenyuk et al., 2006). It is necessary to possess information from many NM stations separated in both longitude and latitude in order to cover a wide range of cut-off rigidities and of viewing directions at the border of the geomagnetosphere. Thus, measurements performed at different cut-off rigidity (geomagnetic latitudes) provide information necessary to derive the SEP spectral characteristics, while NMs over a wide range of latitudes and longitudes are used to assess the SEP anisotropy and the apparent source position. Both spectral and anisotropy characteristics of SEPs are usually obtained simultaneously during an optimization procedure. Note, that the model shall reproduce both, responses of NM stations with statistically significant increases as well as with null or marginal increases, the latter being important to assess the boundary characteristics of the SEP spectra and anisotropy.

In this study we employ a method developed on the basis of previous models (Cramp et al., 1997; Vashenyuk et al., 2006), the details are given elsewhere (Mishev et al., 2014; Mishev and Usoskin, 2016a). The analysis of GLEs using NM data consists of several consecutive steps: (1) computation of asymptotic viewing cones and cut-off rigidity of the selected NMs by simulation of particle propagation in the magnetosphere; (2) making an initial guess of the optimization procedure (inverse problem) by assuming the apparent source position along the interplanetary magnetic field (IMF) line and/ or by application of a procedure similarly to Cramp et al. (1995). The IMF direction is derived explicitly from the measurements of the ACE satellite. Moreover, considering the time shift of the IMF direction at the nose of the Earth's bow shock, the measured solar wind speed is being used and the propagation time from L1 point to the Earth is taken into account similarly to Mishev and Usoskin (2016b); Kocharov et al. (2017); (3) application of an optimization procedure using modelled and measured NM response over a selected space of unknowns in order to derive the primary SEP rigidity spectrum and anisotropy characteristics. The relative count rate increase of a given NM is expressed as:

$$
\frac{\Delta N\left(P_{\mathrm{cut}}\right)}{N}=\frac{\int_{P_{\mathrm{cut}}}^{P_{\max }} J_{\| \mathrm{SEP}}(P, t) Y(P) G(\alpha(P, t)) A(P) d P}{\int_{P_{\mathrm{cut}}}^{\infty} J_{\mathrm{GCR}}(P, t) Y(P) d P},
$$

where $J_{\| \mathrm{SEP}}(P, t)$ is the rigidity spectrum of the primary SEPs with a given rigidity $P$ in the direction of the maximal flux along the IMF, $J_{\mathrm{GCR}}(P, t)$ is the rigidity spectrum of GCR at a given time $t$ with the corresponding modulation, $Y(P)$ is the NM yield function, $G(\alpha(P, t))$ is the pitch angle distribution (PAD) of SEPs, the pitch angle $\alpha$ is defined as the angle between the charged particle's velocity vector $\mathbf{V}$ and the local magnetic field direction $\mathbf{M}$ i.e. $\cos (\alpha)=\mathbf{M} \cdot \mathbf{V}, A(P)$ is a discrete function with $A(P)=1$ for allowed trajectories (proton with rigidity $P$ can reach the station), accordingly $A(P)=0$ for forbidden trajectories (proton with rigidity $P$ can't reach the station), the function $A$ is determined during asymptotic cone calculations, $N$ is the count rate due to GCR, $\Delta N\left(P_{\text {cut }}\right)$ is the count rate increase due to SEPs, $P_{\text {cut }}$ is the lower cut-off rigidity of the station, i.e. the rigidity of the last allowed trajectory, below which all trajectories are forbidden, accordingly $P_{\max }$ is the maximal rigidity of SEPs considered in the model to be $20 \mathrm{GV}$, which is sufficiently high for SEPs. The relative increase of the count rate of a NM station represents the ratio between the NM count rates due to SEPs and GCR averaged over two hours before the event's onset. In this study we used a newly computed NM yield function, which considers the finite lateral extend of CR induced atmospheric cascade and which provides a good agreement with experimental latitude surveys and other measurements and models (Mishev et al., 2013; Gil et al., 2015; 


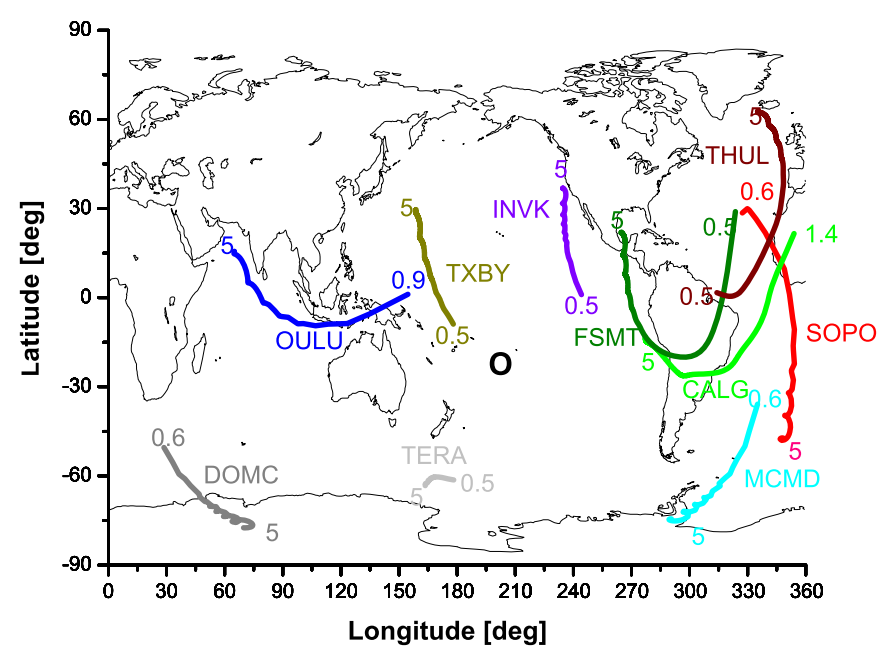

Fig. 2. Computed asymptotic directions for several NM stations during the sub-GLE event on 29 October 2015. The abbreviations (Tab. B1), the corresponding color lines and numbers indicate the NM stations and asymptotic directions, which are plotted in the rigidity range between the cut-off rigidity and $5 \mathrm{GV}$. The small oval corresponds to the direction of the IMF derived from the ACE satellite measurements during the event onset.

Mangeard et al., 2016). Moreover, in order to reduce some model uncertainties, namely the application of two-attenuationlengths method, i.e. normalization of high-altitude NM count rates to the sea level, we have employed NM yield functions for different altitudes (Mishev et al., 2015). The yield function of the mini NM at Dome $\mathrm{C}$ was scaled to a standard 6NM64 according to Caballero-Lopez (2016); Lara et al. (2016). Therefore, the response of each NM is computed with its own yield function corresponding to the exact altitude above the sea level.

In our model the rigidity spectrum of SEPs is described by a modified power law similarly to Cramp et al. (1997); Vashenyuk et al. (2008):

$$
J_{\|}(P)=J_{0} P^{-(\gamma+\delta \gamma(P-1))},
$$

where $J_{\|}(P)$ is the particle flux with given rigidity $P$ in [GV] arriving from the Sun along the axis of symmetry whose direction is defined by geographic coordinate angles $\Psi$ and $\Lambda$ (latitude and longitude), $\gamma$ is the power-law spectral exponent at rigidity $P=1 \mathrm{GV}, \delta \gamma$ is the rate of the spectrum steepening. In equation (1) we can consider also an exponential spectrum similarly to Vashenyuk et al. (2008):

$$
J_{\|}(P)=J_{0} \exp \left(-P / P_{0}\right),
$$

where $J_{\|}$is defined as in equation (2) and $P_{0}$ is a characteristic proton rigidity.

Accordingly, the PAD in both cases is assumed to be a superposition of two Gaussians, which allows to model a bidirectional particle flow:

$$
G(\alpha(P)) \sim \exp \left(-\alpha^{2} / \sigma_{1}^{2}\right)+B \exp \left(-(\alpha-\pi)^{2} / \sigma_{2}^{2}\right),
$$

where $\alpha$ is the pitch angle, $\sigma_{1}$ and $\sigma_{2}$ are parameters corresponding to the width of the $\mathrm{PAD}, \mathrm{B}$ is a parameter

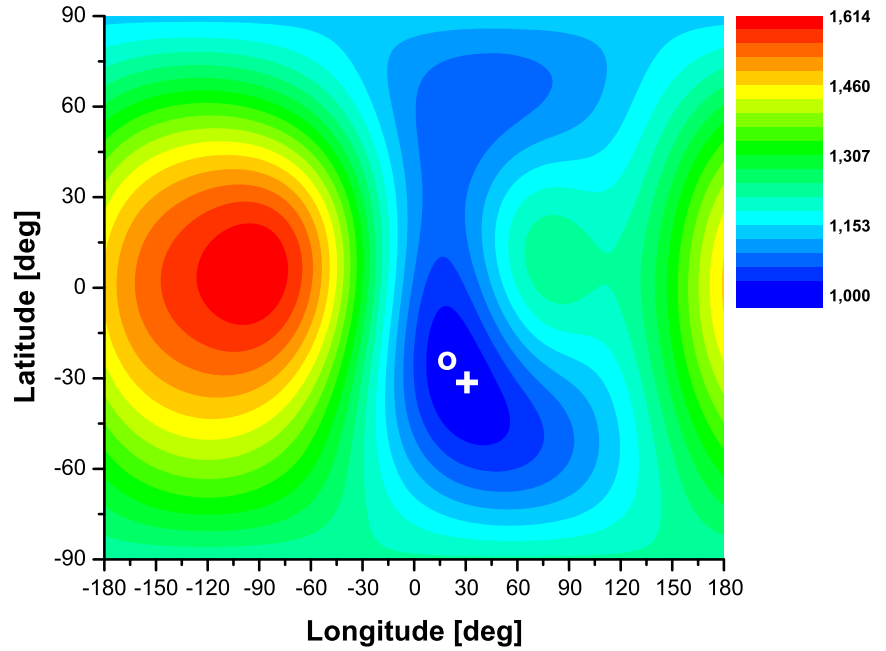

Fig. 3. Contour plot of $D$ (Eq. (6)) for the best fit solutions vs. geographic latitude and longitude during the sub-GLE event on 29 October 2015, normalized to the minimal value of $D$. The cross corresponds to the assumed apparent source position, while the small oval corresponds to the direction of the IMF derived from the ACE satellite measurements during the event onset.

corresponding to the contribution of the particle flux arriving from the anti-sun direction. Therefore, according to equations (1)-equation (4) eight parameters have to be determined $\left(J_{0}, \gamma\right.$, $\left.\delta \gamma, \Psi, \Lambda, \sigma_{1}, \sigma_{2}, B\right)$ for a modified power-law rigidity spectrum (Eq. (2)) or seven $\left(J_{0}, P_{0}, \Psi, \Lambda, \sigma_{1}, \sigma_{2}, B\right)$ for an exponential spectrum (Eq. (3)).

The optimization is performed by minimizing the sum of the squared difference between the modelled and measured $\mathrm{NM}$ responses i.e. minimum of the functional $F$ over the vector of $n$ unknowns and $m$ NM stations:

$$
F=\sum_{i=1}^{m}\left[\left(\frac{\Delta N_{i}}{N_{i}}\right)_{\text {mod. }}-\left(\frac{\Delta N_{i}}{N_{i}}\right)_{\text {meas } .}\right]^{2}
$$

In the study presented here the optimization was performed using the Levenberg-Marquardt method (Levenberg, 1944; Marquardt, 1963) with variable regularization (Tikhonov et al., 1995) and an additional simulation of the NM network response (see Sect. 2.2). The quality of the derived solution is assessed by a combination of several criteria. First, we employed a general criterion, namely the square root of the sum of squared relative difference between the observed and calculated increases for each NM station (residual) normalized to the sum of the measured relative increases (Eq. (6)) (e.g. Himmelblau, 1972).

$$
D=\frac{\sqrt{\sum_{i=1}^{m}\left[\left(\frac{\Delta N_{i}}{N_{i}}\right)_{\text {mod. }}-\left(\frac{\Delta N_{i}}{N_{i}}\right)_{\text {meas } .}\right]^{2}}}{\sum_{i=1}^{m}\left(\frac{\Delta N_{i}}{N_{i}}\right)_{\text {meas. }}},
$$

A good convergence of the optimization process and a robust solution are achieved when $D \leq 5 \%$ (Vashenyuk et al., 2006). This is easy to fulfil for strong events, but hardly possible for weak events. Therefore, we are using an additional 
Table 1. Derived spectral and angular characteristics of sub-GLE events. The columns depict the SEP spectral characteristics, corresponding to the considered sub-GLE. The SP1 and SP2 correspond to SEP with minimal (SP1) and maximal (SP2) differential proton flux at rigidity 1 GV, i. e. the patterns in Figures 5, 7, and 9. The apparent source position geographic coordinates $\Psi$ (latitude) and $\Lambda$ (longitude) are derived with simulation, representing the center of the minimal contour of the sum of variances $D$ (Eq. (6)) for the best fit solutions (see Figs. 3 , A9 and A10).

\begin{tabular}{|c|c|c|c|c|c|c|}
\hline Spectral characteristics & \multicolumn{2}{|c|}{ Sub-GLE 07/03/2012 } & \multicolumn{2}{|c|}{ Sub-GLE 06/01/2014 } & \multicolumn{2}{|c|}{ Sub-GLE 29/10/2015 } \\
\hline$J_{0}\left[\mathrm{~m}^{-2} \mathrm{~s}^{-1} \mathrm{sr}^{-1} \mathrm{GV}^{-1}\right]$ & $4.58 \times 10^{4}$ & $5.55 \times 10^{4}$ & $1.17 \times 10^{4}$ & $1.8 \times 10^{4}$ & $15.8 \times 10^{3}$ & $2.51 \times 10^{4}$ \\
\hline$\gamma$ & 6.1 & 7.2 & 7.28 & 8.15 & 6.0 & 6.82 \\
\hline$\delta \gamma$ & 0 & 0 & 0.2 & 0.2 & 0.1 & 0.12 \\
\hline$\Psi\left[^{\circ}\right]$ & 48 & 48 & -15 & -15 & -30 & -30 \\
\hline$D$ & 11.1 & 9.2 & 6.9 & 7.8 & 13.1 & 12.8 \\
\hline
\end{tabular}

criterion, namely the relative difference between the observed and calculated NM increases must be of the order of about $10 \%$ with a uniform distribution of the residuals i.e. the number of NMs with under and/or over estimation of the count rate must be roughly equal. Note, that this is only a general description of the method, which possess several features and modifications when applied for sub-GLE analysis (see Sect. 2.2).

For the computation of NM cut-off rigidity and asymptotic cones we used the MAGNETOCOSMICS code (Desorgher et al., 2005). We employed a combination of the geomagnetic model IGRF (epoch, 2015) (Macmillan et al., 2003) as the internal field and the Tsyganenko 89 model (Tsyganenko, 1989) for the external field. This combination provides a good balance between simplicity and realism (Kudela and Usoskin, 2004; Nevalainen et al., 2013). For the GCR spectrum we applied a parametrisation based on the force-field model (Gleeson and Axford, 1968; Caballero-Lopez and Moraal, 2004) with a solar modulation parameter calculated according to Usoskin et al. (2011). For the GCRs we considered the nucleonic ratio of heavier particles including $\alpha$-particles to protons in the interstellar medium as 0.3 similarly to Kovaltsov et al. (2012), assuming the local interstellar spectrum according to Burger et al. (2000) and Usoskin et al. (2005).

For a good convergence of the optimization procedure several NM stations with non-null response are necessary (about 2(n-1)) (e.g. Himmelblau, 1972). However, in the case of a sub-GLE event (possible candidates are discussed in Appendix A) all but high-altitude polar NMs have null and/or marginal increase. Therefore, we possessed information from only one or two NMs with a statistically significant increase, but considerably smaller compared to the majority of GLEs. This eventually leads to an ill-posed inverse problem, which requires additional simplifications (see Sect. 2.2) and/or specific numeric procedures (e.g. Tikhonov et al., 1995; Dennis and Schnabel, 1996; Aster et al., 2005).

\subsection{Assessment of spectral and angular constraints of sub-GLE particles}

In order to perform a consistent convergence of the optimization procedure, it is necessary to simplify the model. We assumed a simple power law or exponential rigidity spectrum of SEPs, with one directional Gaussian PAD (without the second term in Eq. (4), namely $B=0$ ) and consider primary particles with vertical incidence only. In addition, we forced the apparent source position to be along the IMF derived from ACE satellite measurements, but not as a free parameter. Therefore, we reduced the number of unknowns to three, namely $J_{0}, \gamma$ and $\sigma$. However, several solutions with similar residual (Eq. (6)) would be derived (Himmelblau, 1972). Subsequently, we considered the mean of those solutions as a likelihood solution, assuming a normal distribution of the derived set of solutions. As the next step, we performed a simulation of the global NM network response with this set of parameters and by fixing the PAD and spectral characteristics, but varying the apparent source position location over all geographic coordinates. The best fit of the global NM network response from this forward modelling is assumed as the final apparent source position, which is subsequently used as an input for the next step, namely optimization procedure using Levenberg-Marquardt method. Note, that the assumed apparent source position (cross) is close to IMF direction (small ovals) on the event(s) onset derived from ACE satellite measurements (Figs. 3, A9, A10). This procedure is repeated in several consecutive iteration steps until the solution quality criteria are achieved.

Thus, by both optimization over a limited set of parameters (e.g. Himmelblau, 1972; Aster et al., 2005) and simulation of the global NM network response (fixing one and/or several of the parameters, but varying the others) after several iterations we assessed the spectral and angular characteristics of subGLE particles and accordingly their spectral constraints. Hence, we derived a set of spectra and PADs and we assessed the spectral and angular characteristics of SEPs with a given confidence level, in our case 95\%. The spectra SP1 and SP2 determine the confidence limit of $95 \%$ of the derived solutions (see Tab. 1 and the corresponding patterns in Figs. 5-10. The spectra SP1 and SP2 correspond to the maximal respectively minimal differential SEP flux at $1 \mathrm{GV}$ in the direction of anisotropy during the event(s). The residual $D$ for each derived SP1 and SP2 for the various events is presented in Table 1.

For moderately strong events it is easy to achieve $D \leq 5 \%$, which leads to a reasonable agreement between the modelled and experimental NM increases (Vashenyuk et al., 2008; 


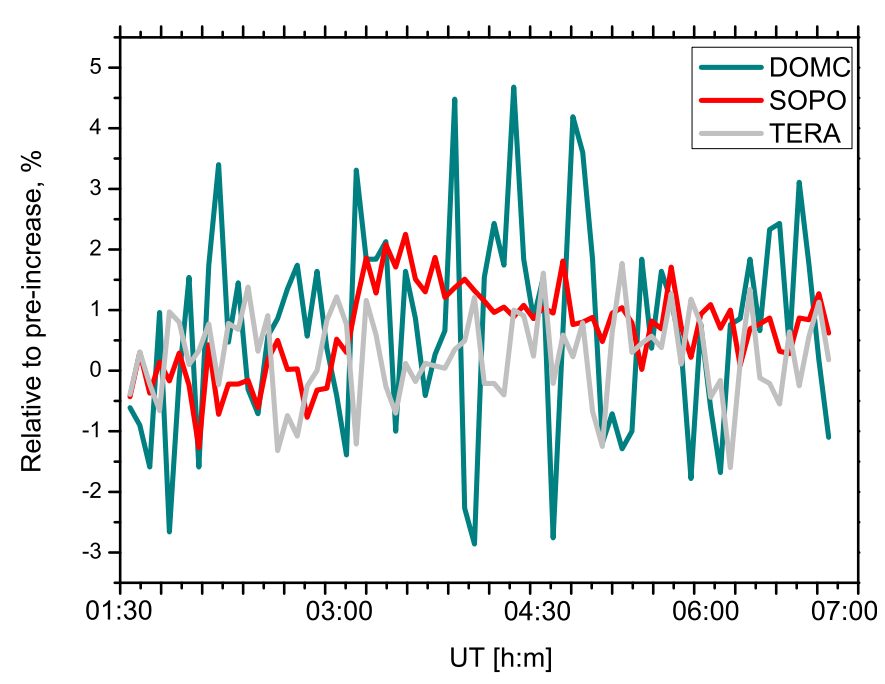

Fig. 4. Time variations of NM count rates with the best response during the sub-GLE event on 29 October 2015 as denoted in the legend. Note the non-uniform time step.

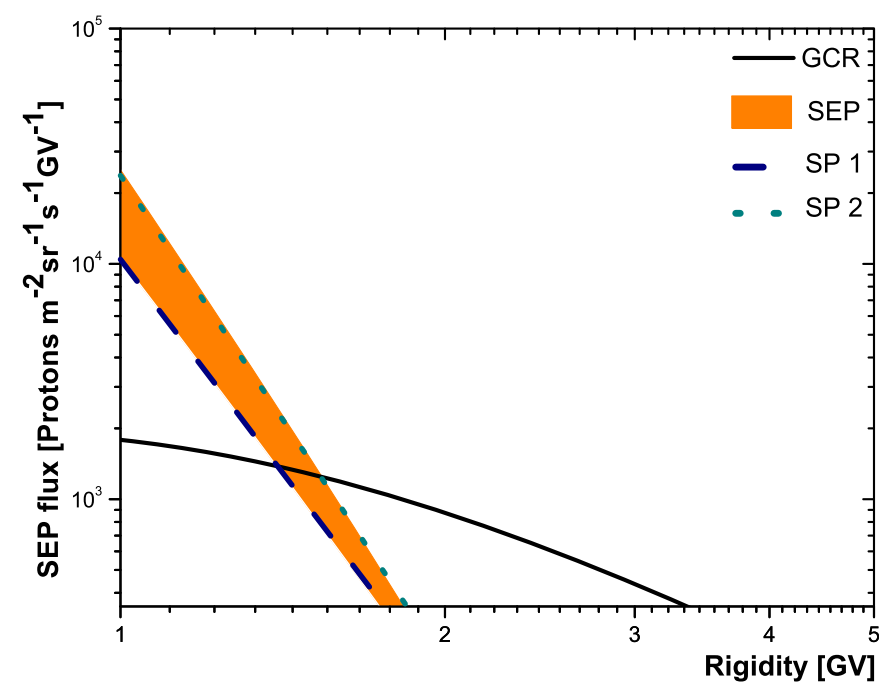

Fig. 5. Derived set of SEP rigidity spectra (orange pattern) during the main phase (02:30-03:30 UT) of sub-GLE event on 29 October 2015. The black solid line denotes the GCR flux, which corresponds to the time period of the sub-GLE occurrence, while SP1 and SP2 correspond to minimal, respectively maximal differential proton flux, the details are given in Table 1.

Mishev and Usoskin, 2016a). However, for weak GLEs or subGLEs $D$ is considerably larger (see Tab. 1). Therefore, the general criterion $D$ (see Eq. (6)) is not the primarily applied (Sect. 2.1). Here, the aim is to achieve about $15-20 \%$ relative difference between measured and modelled NM responses for NM with statistically significant increases, namely high-altitude polar ones, while for all other stations (with marginal and/or null response) it is larger. In order to avoid a normalization to zero, the null responses are assumed to be equal to $0.1 \%$. Hence, we have used a combination of two criteria for best fit solution,

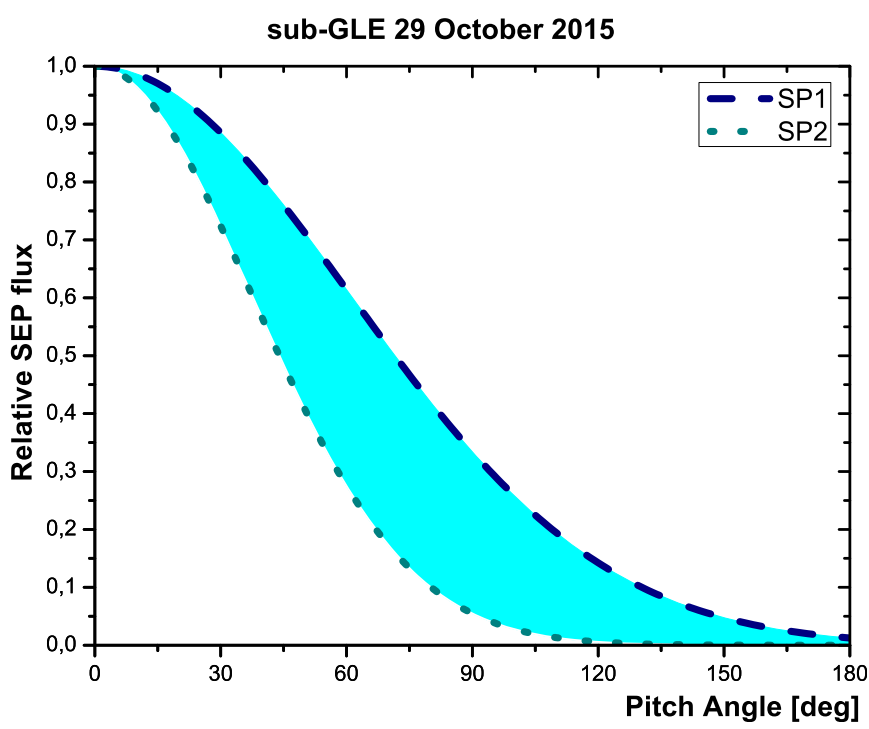

Fig. 6. Derived set of SEP PADs during the main phase (02:30-03:30 UT) of sub-GLE event on 29 October 2015. The details for SP1 and SP2 are given in Table 1.

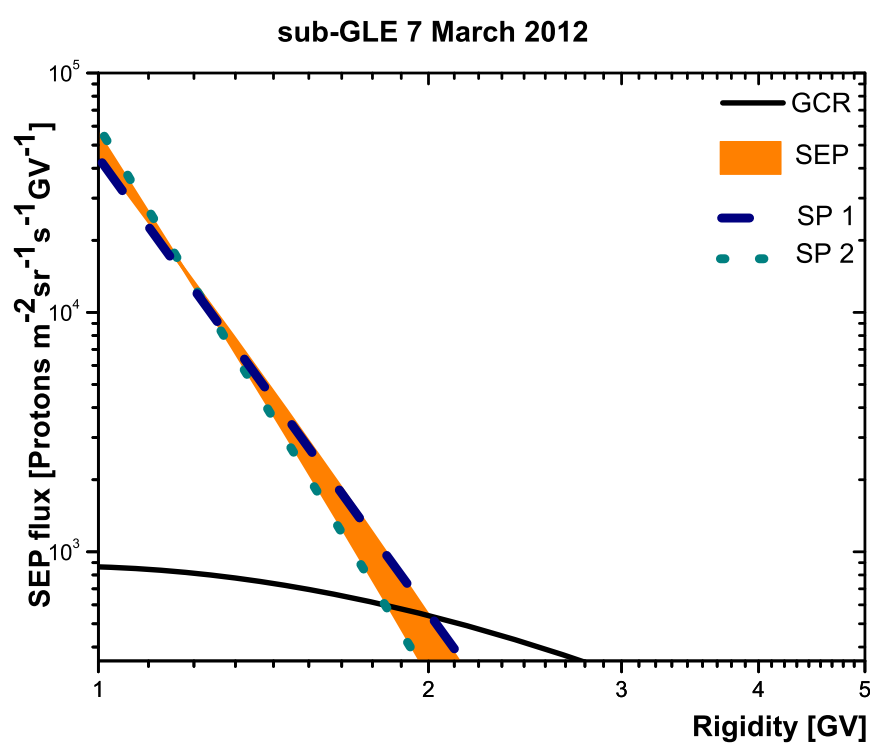

Fig. 7. Derived set of SEP rigidity spectra (orange pattern) during the main phase (09:00-11:00 UT) of sub-GLE event on 7 March 2012. The black solid line denotes the GCR flux, which corresponds to the time period of the sub-GLE occurrence, while SP1 and SP2 correspond to minimal, respectively maximal differential proton flux, the details are given in Table 1 .

namely relative difference between measured and modelled responses of high-altitude polar NMs of about $15-20 \%$ and $D \leq 15 \%$. The residual $D$ is larger than the residual during GLE analysis (e.g. Mishev and Usoskin, 2016a), which results on relative error of the flux in the direction of maximum intensity at $1 \mathrm{GV}$ of about $30-50 \%$ for the different events. The estimated relative error of the flux in the direction of maximum intensity at $1 \mathrm{GV}$ is less than the systematic error of the method for estimation of spectral and angular characteristics of SEPs using 


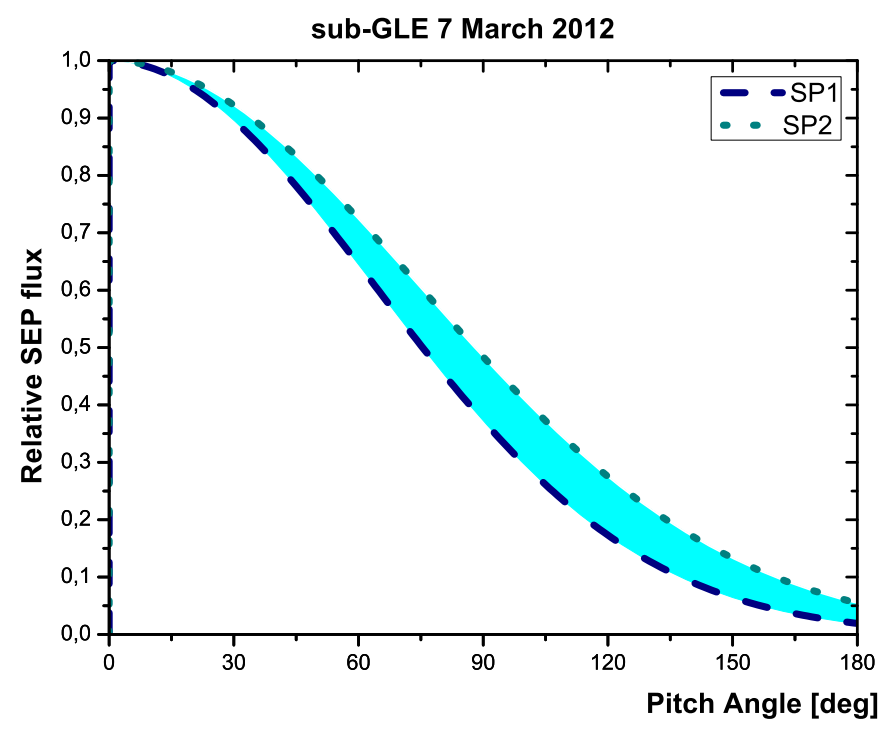

Fig. 8. Derived set of SEP PADs during the main phase (09:00-11:00 UT) of sub-GLE event on 7 March 2012. The details for SP1 and SP2 are given in Table 1.

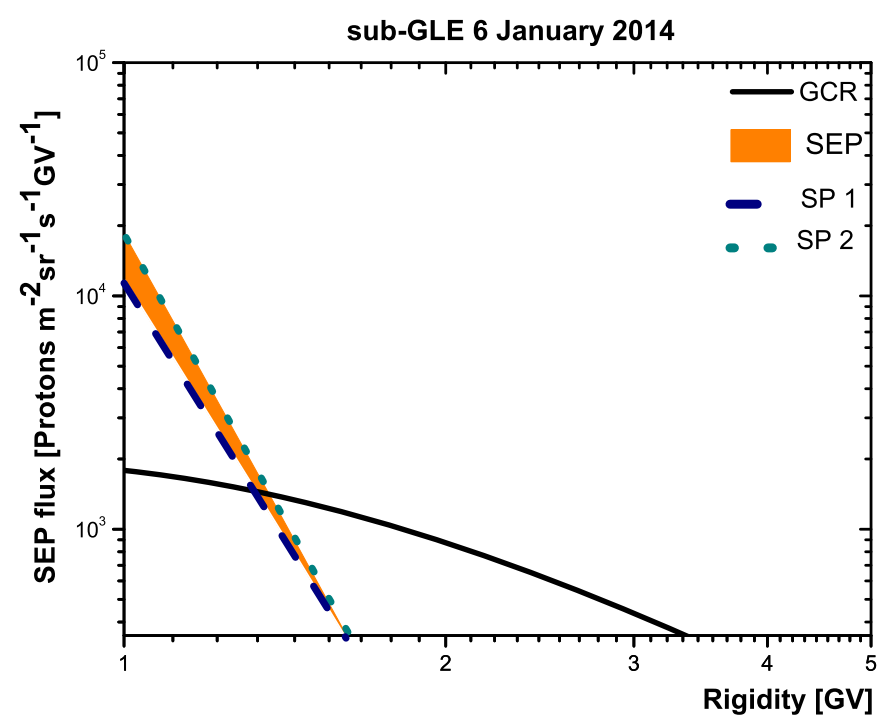

Fig. 9. Derived set of SEP rigidity spectra (orange pattern) during the main phase (08:00-10:00 UT) of sub-GLE event on 6 January 2014. The black solid line denotes the GCR flux, which corresponds to the time period of the sub-GLE occurrence, while SP1 and SP2 correspond to minimal, respectivelymaximal differential proton flux, the details are given in Table 1.

NM data as it was recently discussed in Bütikofer and Flückiger (2013, 2015).

As an example, we demonstrate the analysis of a sub-GLE event on 29 October 2015 (Figs. A5 and A6). Computed asymptotic directions for several NM station locations are shown in Figure 2.

The contour plots of the sum of variances (Eq. (6)) for the best fit solutions obtained by forward modelling of the global NM network response vs. geographic latitude $\Psi$ and longitude $\Lambda$ are presented in Figure 3. The location of the minimal contour of sum of variances normalized to minimal $D$ is

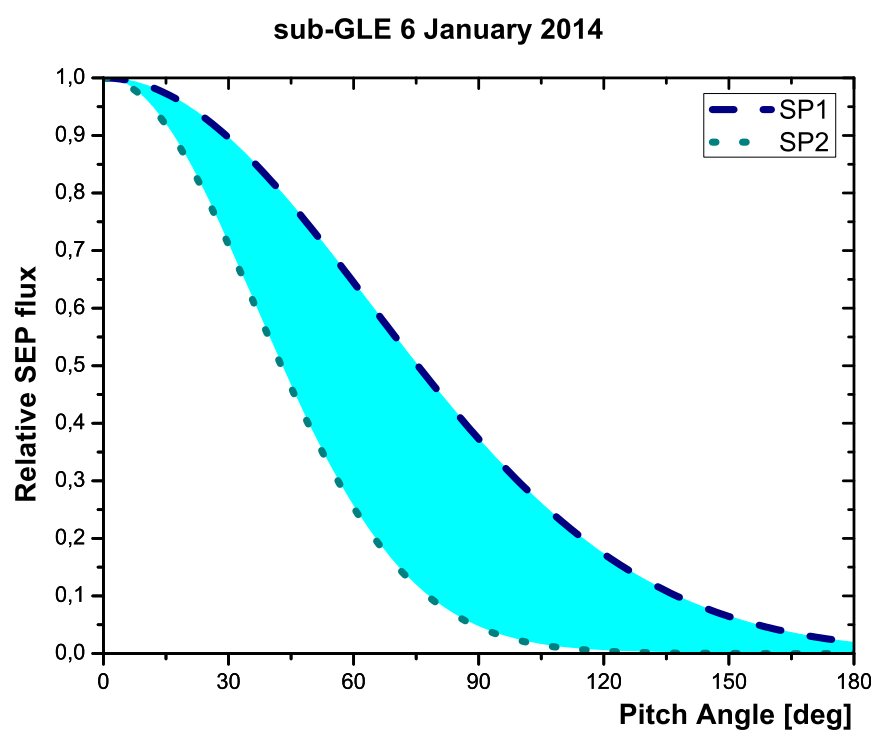

Fig. 10. Derived set of SEP PADs during the main phase (08:0010:00 UT) of sub-GLE event on 6 January 2014. The details for SP1 and SP2 are given in Table 1.

assumed as the apparent source position (see Fig. 3 and Tab. 1) for the subsequent assessment of spectral and angular characteristics of sub-GLE particles by both optimization and simulation of the global NM response.

The optimization and simulation of the global NM network was performed over several stations (the full list of used NM is given in Appendix B). All stations, but South Pole and Dome C NMs had a null response for this event (Figs. 4 and A5). Even Terre Adelie and McMurdo with asymptotic cones close to the assumed apparent source position provided no clear response, because of the higher atmospheric cut-off compared to highaltitude polar NMs. This allowed us to assess the constraints of the derived spectral characteristics (Fig. 5), accordingly angular distributions (Fig. 6). Note, that the modelling of the global NM network response assuming an exponential rigidity spectrum (Eq. (3)) resulted on a considerably larger residual compared to the power law.

Similarly, the spectral and angular characteristics of SEPs are assessed for two other sub-GLE candidates (see Appendix A). The computed NM asymptotic directions during sub-GLE event on 7 March 2012 are presented in Figure A7 and during the event on 6 January 2014 in Figure A8. The corresponding contour plots of the sum of variances for the best fit solutions vs. geographic latitude and longitude are presented in Figure A9 (sub-GLE on 7 March 2012) and Figure A10 (sub-GLE on 6 January 2014). The derived set of SEP rigidity spectra during the main phase of the sub-GLE event on 7 March 2012 is shown in Figure 7, accordingly the PADs in Figure 8.

The assessment of the spectral and angular characteristics of sub-GLE events on 7 March 2012 and on 6 January 2014 is more difficult, because only South Pole NM recorded statistically significant increase (the Dome C NMs were not yet operational). All sea level NMs, including those with asymptotic cones close to the apparent source position (see Figs. A7 and A8), namely McMurdo, Thule, Oulu and Barentsburg (sub GLE on 7 March 2012), respectively Inuvik, McMurdo, Oulu, Thule, Terre Adelie, Fort Smith (sub-GLE 6 
January 2014) provided no clear response, considered as null during the modelling. In addition, the Kingston NM also recorded null increase, because of the higher cut-off rigidity. The final solution is obtained using a similar procedure of consecutive iteration steps by both optimization over a limited set of parameters and a full simulation of the global NM network response (fixing one and/or several of the parameters, but varying the others) and additionally varying the regularization parameter similarly to (e.g. Tikhonov et al., 1995).

The derived set of rigidity spectra during the main phase of sub-GLE event on 6 January 2014 are presented in Figure 9, accordingly PADs in Figure 10.

In general, the assessed rigidity spectra of sub-GLEs are distinctly softer than those of GLEs. Here (Fig. 11) we compare the assessed sub-GLE rigidity spectra with previously derived rigidity spectra of a moderately strong (GLE 70 on13 December 2006) and a weak (GLE 71 on 17 May 2012) GLE events as considered according to recent studies, based on the same approach and methods (Mishev et al., 2014; Mishev and Usoskin, 2016a). The differential proton flux at $1 \mathrm{GV}$ during sub-GLE events is about 4-10 times lower compared to a weak event as GLE 71 (Fig. 11). Note, that in Figure 11 we present the sub-GLE rigidity spectra with maximal flux $J_{0}$, i.e. SP2 from Table 1.

One can see that the assessed SEP rigidity spectra during sub-GLE events are considerably softer than for GLE 70, but similar in the spectral slope to GLE 71. A summary of the assessed spectral and angular characteristics of sub-GLE events is given in Table 1. The SEP spectrum during the subGLE event on 6 January 2014 is consistent with a recent result derived using a space-borne instrument(s) (Kühl et al., 2015). A detailed comparison with space-borne studies (e.g. Tylka and Dietrich, 2009; Sandberg et al., 2014) is planned as forthcoming work.

\section{Application for assessment of ambient dose equivalent at flight altitude}

The radiation environment in the Earth atmosphere can be affected during SEP events (Vainio et al., 2009). The flux of GCR and SEPs is influenced by the spatial-temporal variability of the complex magnetospheric and interplanetary conditions. The radiation environment, accordingly aircrew exposure depends on geographic position, altitude and solar activity (Spurny et al., 1996, 2002; Shea and Smart, 2000). During the SEP events the exposure is a superposition of the GCR and SEPs contributions. It was demonstrated that the aircrew exposure can be estimated on the basis of a full simulation of the atmospheric cascade (Ferrari et al., 2001; Roesler et al., 2002). Several models have been proposed, aiming to estimate the radiation dose rate (effective and/or ambient dose equivalent) at flight altitudes (Schraube et al., 2000; Ferrari et al., 2001; Roesler et al., 2002; Lewis et al., 2005; Sato et al., 2008; Matthiä et al., 2008; Mertens et al., 2013; Mishev et al., 2015). In this study, we employed a recently proposed numerical model for computation of effective and/or ambient dose equivalent at flight altitudes, the details are given elsewhere Mishev and Usoskin (2015). The model demonstrated very good agreement with reference data (Menzel, 2010).

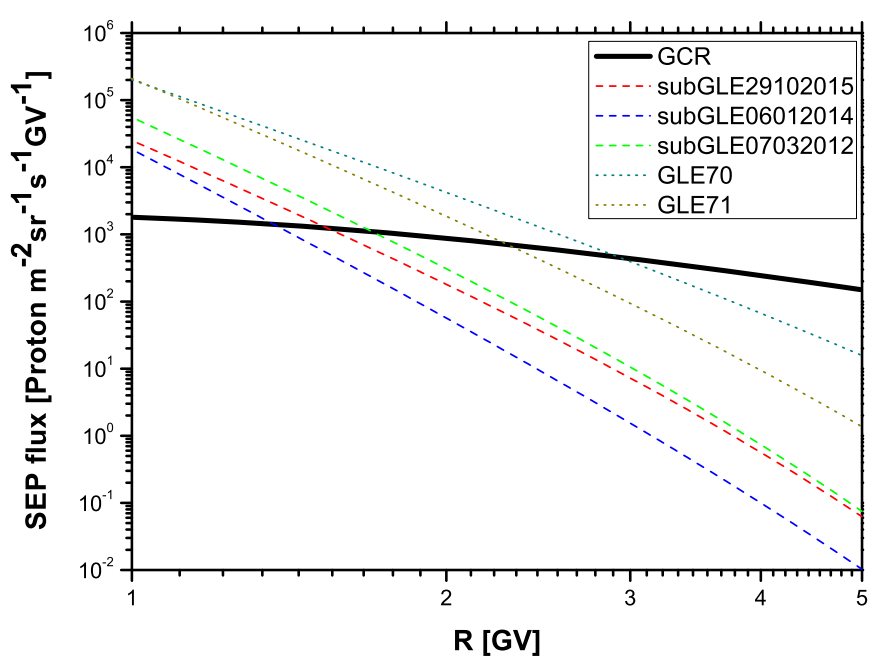

Fig. 11. Rigidity spectra during sub-GLE events compared with GLE 70 on 13 December 2006 and GLE 71 on 17 May 2012 as denoted in the legend. The black solid line in the left panel denotes the average GCR flux.

The model is based on pre-computed effective dose yield functions. The effective dose rate at a given atmospheric depth $h$ induced by a primary CR particle with kinetic energy $T^{\prime}$ is computed using the expression:

$$
E\left(h, T^{\prime}\right)=\sum_{i} \int_{T_{\mathrm{cut}}^{\prime}\left(P_{c}\right)}^{\infty} J_{i}\left(T^{\prime}\right) Y_{i}\left(T^{\prime}, h\right) \mathrm{d} T^{\prime}
$$

where $J_{i}\left(T^{\prime}\right)$ is the differential energy spectrum of the primary $\mathrm{CR}$ arriving at the top of the atmosphere for $i$ component (proton or $\alpha$-particle) and $Y_{i}$ is the effective dose yield function. The integration is over the kinetic energy above $T^{\prime}{ }_{\text {cut }}\left(P_{c}\right)$, which is defined by the local cut-off rigidity $P_{c}$ for a nuclei of type $i$ at a given geographic location by the expression

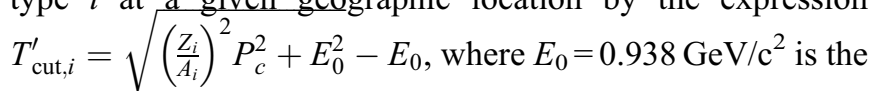
proton's rest mass, $T^{\prime}{ }_{\text {cut }, i}$ is given in $[\mathrm{GeV}]$, respectively $P_{c}$ in [GV].

Accordingly, the effective dose yield function $Y_{i}$ is defined as:

$$
Y_{i}\left(T^{\prime}, h\right)=\sum_{j} \int_{T^{*}} F_{i, j}\left(h, T^{\prime}, T^{*}, \theta, \varphi\right) C_{j}\left(T^{*}\right) d T^{*},
$$

where $C_{j}\left(T^{*}\right)$ is the fluence conversion coefficient of secondary particles of type $j$ (neutron, proton, $\gamma, e^{-}, e^{+}, \mu^{-}, \mu^{+}, \pi^{-}, \pi^{+}$) with energy $T^{*}$ to effective dose, $F_{i, j}\left(h, T^{\prime}, T^{*}, \theta, \varphi\right)$ is the secondary particle fluence of type $j$, produced by a primary particle of type $i$ (proton and/or $\alpha$-particle) with a given primary energy $T^{\prime}$ arriving at the top of the atmosphere from zenith angle $\theta$ and azimuth angle $\varphi$. The conversion coefficients $C_{j}\left(T^{*}\right)$ are considered according to Pelliccioni (2000) and Petoussi-Henss et al. (2010).

With the assessed SEP spectra during the sub-GLE events considered in this study, using equation (7) and the effective dose yield function according to Mishev and Usoskin (2015), 
Table 2. Assessed effective dose rates during maximum phase sub-GLE events at altitude of $35 \mathrm{kft}$ a.s.1. in a region with $P_{c} \leq 1 \mathrm{GV}$. The minimal and maximal effective dose rates are computed using the assessed sub-GLE particles spectra, namely SP1 and SP2 from Table 1. The total effective dose rate is a superposition of the contribution of GCR and sub-GLE particles assuming a maximal value of SEP contribution in order to present a conservative assessment of the exposure.

\begin{tabular}{llll}
\hline Effective dose $E\left(\mu \mathrm{Sv} \cdot \mathrm{h}^{-1}\right)$ & Sub-GLE $07 / 03 / 2012$ & Sub-GLE 06/01/2014 & Sub-GLE 29/10/2015 \\
\hline SEP min & 12.4 & 3.3 & 6.9 \\
SEP max & 14.1 & 4.1 & 8.2 \\
GCR & 5.51 & 5.63 & 5.67 \\
Total & 19.6 & 9.7 & 13.9 \\
\hline
\end{tabular}

we estimated the effective dose rate at a typical commercial flight altitude of about $35 \mathrm{kft}(\approx 11000 \mathrm{~m}$ a.s.1.). For the computations the force field model for GCR spectrum was employed, using the corresponding solar modulation parameter calculated according to Usoskin et al. (2011). We assumed a conservative approach for the sub-GLE particles angular distribution, namely an isotropic distribution. Hence, we estimated the effective dose rate in a region with $P_{c} \leq 1 \mathrm{GV}$, where the expected exposure is maximal. We computed the minimal and maximal effective dose rate due to sub-GLE particles using the SP1 and SP2 from Table 1, respectively. Subsequently, we considered the maximal value for the summation with GCR contribution in order to perform a conservative assessment of the exposure. The computations are summarized in Table 2. One can see that the contribution of SEPs during sub-GLE event is at least comparable to the contribution of GCR. In general, the sub-GLE particles would double the exposure due to GCR. A detailed study for several altitudes, considering explicitly the anisotropy of the events, as well as the dynamical evolution of the sub-GLE particles spectral and angular characteristics throughout the events similarly to Mishev and Usoskin (2015) is planned for forthcoming work.

\section{Conclusions}

In the work presented here we have studied several candidates for a new subclass of SEP events - sub-GLEs, which are of special interest for space weather applications. On the basis of data retrieved from different NMs and using a combination of a full simulation of the global NM network response and optimization procedure over a set of unknown parameters describing the SEP features, we have assessed the spectral and angular characteristics of sub-GLE particles. With the estimated spectral characteristics and using a previously developed model, we have assessed the effective dose rate in a polar and sub-polar region at commercial flight altitudes of $35 \mathrm{kft}$. During those computations a conservative scenario concerning the contribution of sub-GLE to the exposure was assumed. It was shown that the contribution of sub-GLE particles to the exposure is at least comparable to the GCRs contribution. Thus, we demonstrated that the global NM network is a useful tool to estimate an important space weather effect, namely the exposure of aircrew due to $\mathrm{CR}$ of galactic and solar origin.
Acknowledgments. This work was supported by the Academy of Finland (project 272157, Center of Excellence ReSoLVE), projects CRIPA and CRIPA-X No. 304435 and Finnish Antarctic Research Program (FINNARP). The authors are warmly thankful to Askar Ibragimov for GLE database support. The authors acknowledge the NMDB (nmdb.eu) as well as all the PIs of the NM stations used in the study. The authors and the editor thank two anonymous referees for their assistance in evaluating this paper.

\section{References}

Adriani O, et al. 2016. Measurements of cosmic-ray hydrogen and helium isotopes with the PAMELA experiment. Astrophys $J \mathbf{8 1 8}$ : 68. DOI:10.3847/0004-637X/818/1/68.

Aguilar M, et al. 2010. Relative composition and energy spectra of light nuclei in cosmic rays: results from AMS-01. Astrophys $J \mathbf{7 2 4}$ : 329-40, DOI:10.1088/0004-637X/724/1/329.

Aster R, Borchers B, Thurber CH. 2005. Parameter estimation and inverse problems. New York: Elsevier, ISBN 0-12- 065604-3.

Atwell W, et al. 2017. Atmospheric radiation measurement system for commercial aircraft altitudes. In: 9th AIAA Atmospheric and Space Environments Conference, AIAA AVIATION Forum (AIAA 2017-3063), DOI:10.2514/6.2017-3063.

Atwell W, Tylka A, Dietrich W, Rojdev K, Matzkind C. 2015. SubGLE solar particle events and the implications for lightly-shielded systems flown during an era of low solar activity. In: 45th International Conference on Environmental Systems, 12-16 July 2015. Bellevue, WA: ICES-2015-340, pp. 1-12.

Balanov M, et al. 2008. UNSCEAR 2008 Report to the General Assembly with Scientific Annexes Volume II Scientific Annexe B. Tech. rep.

Bazilevskaya GA. 2005. Solar cosmic rays in the near Earth space and the atmosphere. Adv Space Res 35: 458-464, DOI:10.1016/j. asr.2004.11.019.

Bazilevskaya GA, et al. 2008. Cosmic ray induced ion production in the atmosphere. Space Sci Rev 137: 149-173, DOI:10.1007/ s11214-008-9339-y.

Bieber J, Evenson P. 1995. Spaceship Earth - an optimized network of neutron monitors. In: Proc. of 24th ICRC Rome, Italy, 28 August-8 September 1995, vol. 4, pp. 1316-1319.

Bieber J, Clem J, Evenson P, Oh S, Pyle R. 2013, Continued decline of South Pole neutron monitor counting rate. J Geophys Res: Space Phys 118: 6847-6851, DOI:10.1002/2013JA018915.

Bombardieri D, Duldig M, Michael K, Humble J. 2006. Relativistic proton production during the 2000 July 14 solar event: the case for 
multiple source mechanisms. Astrophys $J$ 644: 565-574, DOI: $10.1086 / 501519$.

Burger R, Potgieter M, Heber B. 2000. Rigidity dependence of cosmic ray proton latitudinal gradients measured by the Ulysses spacecraft: implication for the diffusion tensor. J Geophys Res 105: 2744727455, DOI:10.1029/2000JA000153.

Bütikofer R, Flückiger E. 2013. Differences in published characteristics of GLE60 and their consequences on computed radiation dose rates along selected flight paths. J Phys: Conf Ser 409: 012166, DOI:10.1088/1742-6596/409/1/012166.

Bütikofer R, Flückiger E. 2015, What are the causes for the spread of GLE parameters deduced from NM data? J Phys: Conf Ser 632: 012053. DOI:10.1088/1742-6596/632/1/012053.

Caballero-Lopez R. 2016. An estimation of the yield and response functions for the mini neutron monitor. J Geophys Res A: Space Phys 121: 7461-7469, DOI:10.1002/2016JA022690.

Caballero-Lopez R, Moraal H. 2004. Limitations of the force field equation to describe cosmic ray modulation. $J$ Geophys Res 109: A01101. DOI:10.1029/2003JA010098.

Carmichael H. 1968. Cosmic rays (instruments). In: Minnis CM, ed. Ann. IQSY. Cambridge, MA: MIT Press, vol. 1, pp. 178-197.

Carmichael H, Bercovitch M, Shea MA, Magidin M, Peterson RW. 1968. Attenuation of neutron monitor radiation in the atmosphere. Can J Phys 46: 1006.

Cliver E, Kahler S, Reames D. 2004. Coronal shocks and solar energetic proton events. Astrophys J 605: 902-910, DOI:10.1086/382651.

Cramp J, Humble J, Duldig M. 1995. The cosmic ray ground-level enhancement of 24 October 1989. In: Proceedings Astronomical Society of Australia, vol. 11, pp. 28-32.

Cramp J, Duldig M, Flückiger E, Humble J, Shea M, Smart D. 1997. The October 22, 1989, solar cosmic ray enhancement: an analysis the anisotropy spectral characteristics. J Geophys Res 102: 2423724248, DOI:10.1029/97JA01947.

Debrunner H, Flückiger E, Gradel H, Lockwood J, McGuire R. 1988. Observations related to the acceleration, injection, and interplanetary propagation of energetic protons during the solar cosmic ray event on February 16, 1984. J Geophys Res 93: 7206-7216, DOI:10.1029/JA093iA07p07206.

Dennis J, Schnabel R. 1996. Numerical methods for unconstrained optimization and nonlinear equations. Englewood Cliffs: PrenticeHall, ISBN 13-978-0-898713-64-0.

Desai M, Giacalone J. 2016. Large gradual solar energetic particle events. Living Rev Sol Phys 13: 3, DOI:10.1007/s41116-016-0002-5.

Desorgher L, Flückiger E, Gurtner M, Moser M, Bütikofer R. 2005. A Geant 4 code for computing the interaction of cosmic rays with the earth's atmosphere. Int J Mod Phys A 20: 6802-6804, DOI:10.1142/S0217751X05030132.

Desorgher L, Kudela K, Flückiger E, Bütikofer R, Storini M, Kalegaev V. 2009. Comparison of Earth's magnetospheric magnetic field models in the context of cosmic ray physics. Acta Geophys 57: 75-87, DOI:10.2478/s11600-008-0065-3.

Dorman L. 2004. Cosmic rays in the Earth's atmosphere and underground. Dordrecht: Kluwer Academic Publishers, ISBN 1-4020-2071-6.

Dorman L. 2006. Cosmic ray interactions, propagation, and acceleration in space plasmas. Astrophysics and space science library. Dordrecht: Springer, vol. 339, ISBN 13-978-1-4020-5100-5.

Eisenbud M, Gesell T. 1997. Environmental radioactivity from natural, industrial and military sources. Academic Press, San Diego, ISBN-13:978-0-12-235154-9.

EURATOM 1996. Council Directive 96/29/EURATOM of 13 May 1996 laying down basic safety standards for protection of the health of workers and the general public against the dangers arising from ionising radiation. Official Journal of the European Communities, 39.

Ferrari A, Pelliccioni M, Rancati T. 2001. Calculation of the radiation environment caused by galactic cosmic rays for determining air crew exposure. Radiat Prot Dosim 93: 101-114, DOI:10.1093/ oxfordjournals.rpd.a006418.

Gaisser TK, Stanev T. 2010. Cosmic rays. In K.N. et al., ed., Review of particle physics. J Phys G 37: 269-275.

Gil A, Usoskin I, Kovaltsov G, Mishev A, Corti C, Bindi V. 2015. Can we properly model the neutron monitor count rate? J Geophys Res 120: 7172-7178, DOI:10.1002/2015JA021654.

Gleeson L, Axford W. 1968. Solar modulation of galactic cosmic rays. Astrophys $J$ 154, 1011-1026.

Grieder P. 2001. Cosmic rays at Earth researcher's reference manual and data book. Amsterdam: Elsevier Science, ISBN 978-0-44450710-5.

Hatton C. 1971. The neutron monitor. In: Progress in elementary particle and cosmic-ray physics. Amsterdam: North Holland Publishing Co., vol. X, chap. 1.

Hatton C, Carmichael H. 1964. Experimental investigation of the NM-64 neutron monitor. Can J Phys 42: 2443-2472.

Heber B, Galsdorf D, Gieseler J, Herbst K, Walther M, Stoessl A, Krüger H, Moraal H, Benadé G. 2015. Mini neutron monitor measurements at the Neumayer III station and on the German research vessel Polarstern. In: Proceedings of Science, Proc. of 34th ICRC Hague, Netherlands, 30 July-6 August 2015, p. 122.

Himmelblau D. 1972. Applied nonlinear programming. McGraw-Hill (Tx), ISBN 978-0070289215.

Humble J, Duldig M, Smart D, Shea M. 1991. Detection of 0.5-15 $\mathrm{GeV}$ solar protons on 29 September 1989 at Australian stations. Geophys Res Lett 18: 737-740.

ICRP 1991. ICRP Publication 60: 1990 Recommendations of the International Commission on Radiological Protection. Ann ICRP 21.

Kocharov L, et al. 2017. Investigating the origins of two extreme solar particle events: proton source profile and associated electromagnetic emissions. Astrophys $J$ 839: 79, DOI:10.3847/1538-4357/ aa6a13.

Kovaltsov G, Mishev A, Usoskin I. 2012. A new model of cosmogenic production of radiocarbon ${ }^{14} C$ in the atmosphere. Earth Planet Sci Lett 337: 114-120, DOI:10.1016/j. eps1.2012.05.036.

Krüger H, Moraal H. 2013. Neutron monitor calibrations: progress report. J Phys: Conf Ser 409: 012171, DOI:10.1088/1742-6596/ 409/1/012171.

Krüger H, Moraal H, Nel R, Krüger H, O’Kennedy M. 2015. The mini neutron monitor programme. In: Proceedings of Science, Proc. of 34th ICRC Hague, Netherlands, 30 July-6 August 2015, p. 223.

Kudela K. 2016. On low energy cosmic rays and energetic particles near Earth. Contrib Astron Obs Skaln Pleso 46: 15-70.

Kudela K, Usoskin I. 2004. On magnetospheric transmissivity of cosmic rays. Czechoslov J Phys 54: 239-254.

Kühl P, Banjac S, Dresing N, Goméz-Herrero R, Heber B, Klassen A, Terasa C. 2015. Proton intensity spectra during the solar energetic particle events of May 17, 2012 and January 6, 2014. Astron Astrophys 576: A120, DOI:10.1051/0004-6361/201424874.

Kühl P, Dresing N, Heber B, Klassen A. 2017. Solar energetic particle events with protons above $500 \mathrm{MeV}$ between 1995 and 2015 measured with SOHO/EPHIN. Sol Phys 292: 10, DOI:10.1007/ s11207-016-1033-8.

Lara A, Borgazzi A, Caballero-Lopez R. 2016. Altitude survey of the galactic cosmic ray flux with a Mini Neutron Monitor. Adv Space Res 58: 1441-1451, DOI:10.1016/j.asr.2016.06.021. 
Lee J, Nam U-W, Pyo J, Kim S, Kwon Y-J, Lee J, Park I, Kim M-H, Dachev T. 2015. Short-term variation of cosmic radiation measured by aircraft under constant flight conditions. Space Weather 13: 797-806, DOI:10.1002/2015SW001288.

Levenberg K. 1944. A method for the solution of certain non-linear problems in least squares. Q Appl Math 2: 164-168.

Lewis B, Bennett L, Green A, Butler A, Desormeaux M, Kitching F, McCall M, Ellaschuk B, Pierre M. 2005. Aircrew dosimetry using the Predictive Code for Aircrew Radiation Exposure (PCAIRE). Radiat Prot Dosim 116: 320-326.

Li C, Miroshnichenko L, Sdobnov V. 2016. Small ground-level enhancement of 6 January 2014: acceleration by CME-driven shock? Sol Phys 291: 975-987, DOI:10.1007/s11207-016-0871-8.

Lilensten J, Bornarel J. 2009. Space weather, environment and societies. Dordrecht: Springer, ISBN 978-1-4020- 4332-1.

Lockwood JA, Debrunner H, O. Flükiger E. 1990. Indications for diffusive coronal shock acceleration of protons in selected solar cosmic ray events. J Geophys Res: Space Phys 95: 4187-4201.

Macmillan S, et al. 2003. The 9th-generation international geomagnetic reference field. Geophys J Int 155: 1051-1056, DOI:10.1016/j.pepi.2003.09.002.

Mangeard P-S, Ruffolo D, Saiz A, Nuntiyakul W, Bieber J, Clem J, Evenson P, Pyle R, Duldig M, Humble J. 2016. Dependence of the neutron monitor count rate and time delay distribution on the rigidity spectrum of primary cosmic rays. J Geophys Res: Space Phys 121: 11620-11636, DOI:10.1002/2016JA023515.

Marquardt D. 1963. An algorithm for least-squares estimation of nonlinear parameters. SIAM J Appl Math 11: 431-441.

Matthiä D, Sihver L, Meier M. 2008. Monte-Carlo calculations of particle fluences and neutron effective dose rates in the atmosphere. Radiat Prot Dosim 131: 222-228, DOI:10.1093/rpd/ncn130.

Mavromichalaki H, et al., 2011 Applications and usage of the realtime Neutron Monitor Database. Adv Space Res 47: 2210-2222, DOI:10.1016/j.asr.2010.02.019.

Menzel H. 2010. The international commission on radiation units and measurements. J ICRU 10: 1-35.

Mertens C, Meier M, Brown S, Norman R, Xu X. 2013. NAIRAS aircraft radiation model development, dose climatology, and initial validation. Space Weather 11: 603-635, DOI:10.1002/swe.20100.

Mishev A, Usoskin I. 2013. Computations of cosmic ray propagation in the Earth's atmosphere, towards a GLE analysis. J Phys: Conf Ser 409: 012152, DOI:10.1088/1742-6596/409/1/012152.

Mishev A, Usoskin I. 2015. Numerical model for computation of effective and ambient dose equivalent at flight altitudes: application for dose assessment during GLEs. J Space Weather Space Clim 5: A10, DOI:10.1051/swsc/2015011.

Mishev A, Usoskin I. 2016a. Analysis of the ground level enhancements on 14 July 2000 and on 13 December 2006 using neutron monitor data. Sol Phys 291: 1225-1239, DOI:10.1007/ s11207-016-0877-2.

Mishev A, Usoskin I. 2016b. Erratum to: Analysis of the ground level enhancements on 14 July 2000 and on 13 December 2006 using neutron monitor data. Sol Phys 291: 1579-1580, DOI:10.1007/ s11207-016-0877-2.

Mishev A, Usoskin I, Kovaltsov G. 2013. Neutron monitor yield function: new improved computations. $J$ Geophys Res 118: 2783-2783, DOI:10.1002/jgra.50325.

Mishev A, Kocharov L, Usoskin I. 2014. Analysis of the ground level enhancement on 17 May 2012 using data from the global neutron monitor network. J Geophys Res 119: 670-679, DOI:10.1002/ 2013JA019253.

Mishev A, Usoskin I, Kovaltsov G. 2015. New neutron monitor yield function computed at several altitudes above the sea level: application for GLE analysis. In: Proceedings of Science, Proc. of 34th ICRC Hague, Netherlands, 30 July-6 August 2015, p. 159.

Mishev A, Adibpour F, Usoskin I, Felsberger E. 2015. Computation of dose rate at flight altitudes during ground level enhancements no. 69, 70 and 71. Adv Space Res 55: 354-362, DOI:10.1016/j. asr.2014.06.020.

Moraal H. 1976. Observations of the eleven-year cosmic-ray modulation cycle. Space Sci Rev 19: 845-920.

Moraal H, Belov A, Clem J. 2000. Design and co-ordination of multistation international neutron monitor networks. Space Sci Rev $\mathbf{9 3}$ 285-303.

Nevalainen J, Usoskin I, Mishev A. 2013. Eccentric dipole approximation of the geomagnetic field: application to cosmic ray computations. Adv Space Res 52: 22-29, DOI:10.1016/j. asr.2013.02.020.

Pelliccioni M. 2000. Overview of fluence-to-effective dose and fluence-to-ambient dose equivalent conversion coefficients for high energy radiation calculated using the FLUKA code. Radiat Prot Dosim 88: 279-297.

Petoussi-Henss N, Bolch W, Eckerman K, Endo A, Hertel N, Hunt J, Pelliccioni M, Schlattl H, Zankl M. 2010. Conversion coefficients for radiological protection quantitiesfor external radiation exposures. Ann ICRP 40: 1-257.

Poluianov S, Usoskin I, Mishev A, Moraal H, Krüger H, Casasanta G, Traversi R, Udisti R. 2015. Mini neutron monitors at Concordia research station, Central Antarctica. J Astron Space Sci 32: $281-$ 287, DOI:10.5140/JASS.2015.32.4.281.

Poluianov S, Usoskin I, Mishev A, Smart D, Shea M. 2017. Revisited definition of GLE. In: Proceedings of Science, Proc. of 35th ICRC Busan, Korea, 12-17 July 2017, p. 125.

Reames D. 1999. Particle acceleration at the Sun and in the heliosphere. Space Sci Rev 90: 413-491.

Reames D. 2013. The two sources of solar energetic particles. Space Sci Rev 175: 53-92, DOI:10.1007/s11214-013-9958-9.

Roesler S, Heinrich W, Schraube H. 2002. Monte Carlo calculation of the radiation field at aircraft altitudes. Radiat Prot Dosim 98: 367 388 .

Sandberg I, Jiggens P, Heynderickx D, Daglis I. 2014. Cross calibration of NOAA GOES solar proton detectors using corrected NASA IMP-8/GME data. Geophys Res Lett 41: 4435-4441, DOI:10.1002/2014GL060469.

Sato T, Yasuda H, Niita K, Endo A, Sihver L. 2008. Development of PARMA: PHITS-based analytical radiation model in the atmosphere. Radiat Res 170: 244-259, DOI:10.1667/RR1094.1.

Schraube H, Leuthold G, Heinrich W, Roesler S, Combecher D. 2000. European program package for the calculation of aviation route doses, version 3.0. Tech. Rep. D-85758. Neuherberg, Germany: National Research Center for Environment and Health Institute of Radiation Protection.

Shea M, Smart D. 1982. Possible evidence for a rigidity-dependent release of relativistic protons from the solar corona. Space Sci Rev 32: 251-271.

Shea M, Smart D. 1990. A summary of major solar proton events. Sol Phys 127: 297-320.

Shea M, Smart D. 2000. Cosmic ray implications for human health. Space Sci Rev 93: 187-205, DOI:10.1023/A:1026544528473.

Simpson J. 1957. Cosmic-radiation neutron intensity monitor. Ann. Int. Geophys. Year, 4: 351-373.

Simpson J, Fonger W, Treiman S. 1953. Cosmic radiation intensitytime variation and their origin. I. Neutron intensity variation method and meteorological factors. Phys Rev, 90: 934-950.

Spurny F, Votockova I, Bottollier-Depois J. 1996. Geographical influence on the radiation exposure of an aircrew on board a subsonic aircraft. Radioprotection 31: 275-280. 
Spurny F, Dachev T, Kudela K. 2002. Increase of onboard aircraft exposure level during a solar flare. Nucl Energy Saf 10: 396-400.

Stoker P. 1995. Relativistic solar proton events. Space Sci Rev 73: 327-385, DOI:10.1007/BF00751240.

Stoker P, Dorman L, Clem J. 2000. Neutron monitor design improvements. Space Sci Rev 93: 361-380.

Thakur N, Gopalswamy N, Xie H, Yashiro S, Akiyama S, Davila J. 2014. Ground level enhancement in the 2014 January 6 solar energetic particle event. Astrophys J Lett 790: L13, DOI:10.1088/ 2041-8205/790/1/L13.

Tikhonov AN, Goncharsky AV, Stepanov VV, Yagola AG. 1995. Numerical methods for solving ill-posed problems. Dordrecht: Kluwer Academic Publishers, ISBN 978-90-481- 4583-6.

Tsyganenko N. 1989. A magnetospheric magnetic field model with a warped tail current sheet. Planet Space Sci 37: 5-20.

Tylka A, Dietrich W. 2009. A new and comprehensive analysis of proton spectra in ground-level enhanced (GLE) solar particle events. In: Proc. of 31st ICRC Lodz, Poland, 7-15 July 2009, p. 0273.

Usoskin I, Kovaltsov G. 2006. Cosmic ray induced ionization in the atmosphere: full modeling and practical applications. $J$ Geophys Res 111: D12206. DOI:10.1029/2006JD007150.

Usoskin I, Alanko-Huotari K, Kovaltsov G, Mursula K. 2005. Heliospheric modulation of cosmic rays: monthly reconstruction for 1951-2004. J Geophys Res 110: A12108. DOI:10.1029/ 2005JA011250.
Usoskin IG, Desorgher L, Velinov P, Storini M, Flückiger E, Bütikofer R, Kovaltsov G. 2009. Ionization of the Earth's atmosphere by solar and galactic cosmic rays. Acta Geophys 57: 88-101, DOI:10.2478/s11600-008-0019-9.

Usoskin I, Bazilevskaya G, Kovaltsov G. 2011. Solar modulation parameter for cosmic rays since 1936 reconstructed from groundbased neutron monitors and ionization chambers. $J$ Geophys Res 116: A02104. DOI:10.1029/2010JA016105.

Usoskin I, Ibragimov A, Shea M, Smart D. 2015. Database of ground level enhancements (GLE) of high energy solar proton events. In: Proceedings of Science, Proc. of 34th ICRC Hague, Netherlands, 30 July-6 August 2015, p. 054.

Vainio R, et al., 2009. Dynamics of the Earth's particle radiation environment. Space Sci Rev 147: 187-231, DOI:10.1007/ s11214-009-9496-7.

Vashenyuk E, Balabin Y, Perez-Peraza J, Gallegos-Cruz A, Miroshnichenko L. 2006. Some features of the sources of relativistic particles at the Sun in the solar cycles 21-23. Adv Space Res 38: 411-417, DOI:10.1016/j.asr.2005.05.012.

Vashenyuk E, Balabin Y, Stoker P. 2007. Responses to solar cosmic rays of neutron monitors of a various design. Adv Space Res 40: 331-337, DOI:10.1016/j.asr.2007.05.018.

Vashenyuk E, Balabin Y, Gvozdevsky B, Schur L. 2008. Characteristics of relativistic solar cosmic rays during the eventof December 13, 2006. Geomagn Aeron 48: 149-153, DOI:10.1007/s11478-0082003-6.

Cite this article as: Mishev A, Poluianov S, Usoskin I. 2017. Assessment of spectral and angular characteristics of sub-GLE events using the global neutron monitor network. J. Space Weather Space Clim. 7: A28 


\section{Appendices}

\section{Appendix A Candidates for sub-GLE events}

Candidates for sub-GLEs should exceed SEPs energy of about $300 \mathrm{MeV} /$ nucleon (Atwell et al., 2015; Kühl et al., 2017). There are several candidates for sub-GLE events, now included in the GLE database (gle.oulu.fi) (Usoskin et al., 2015). According to the definition (e.g. Poluianov et al., 2017), a subGLE event is registered if a statistically significant increase is observed by at least two differently located high-altitude NM stations, but without a response of near sea level NMs. This definition requires the registration of the SEP event by one and/or two high-altitude polar NM stations, namely South Pole and Dome C.

The first candidate event was observed on 7 March 2012. The event occurred during a period of active Sun and during disturbed geomagnetospheric conditions. A non-null response was observed only by the South Pole NMs (Fig. A1). The South Pole NMs signal was accompanied with an increase of the proton flux in the GOES 13 data (Fig. A2). This event is a candidate, since it was not observed by any other NM (Dome C NMs were not operational yet). However, according to our estimations considering the assessed spectral and angular characteristics (see Tab. 1 and Figs. 7 and 8) it would be

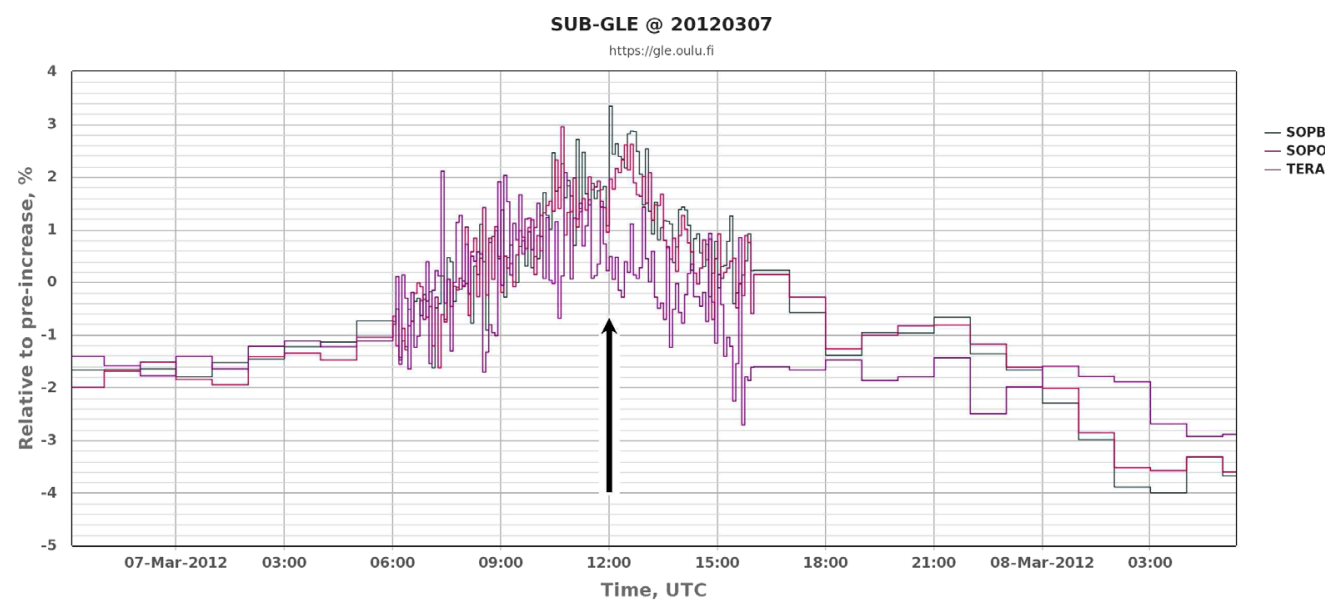

(a) NMs with statistically significant increase compared with TERA

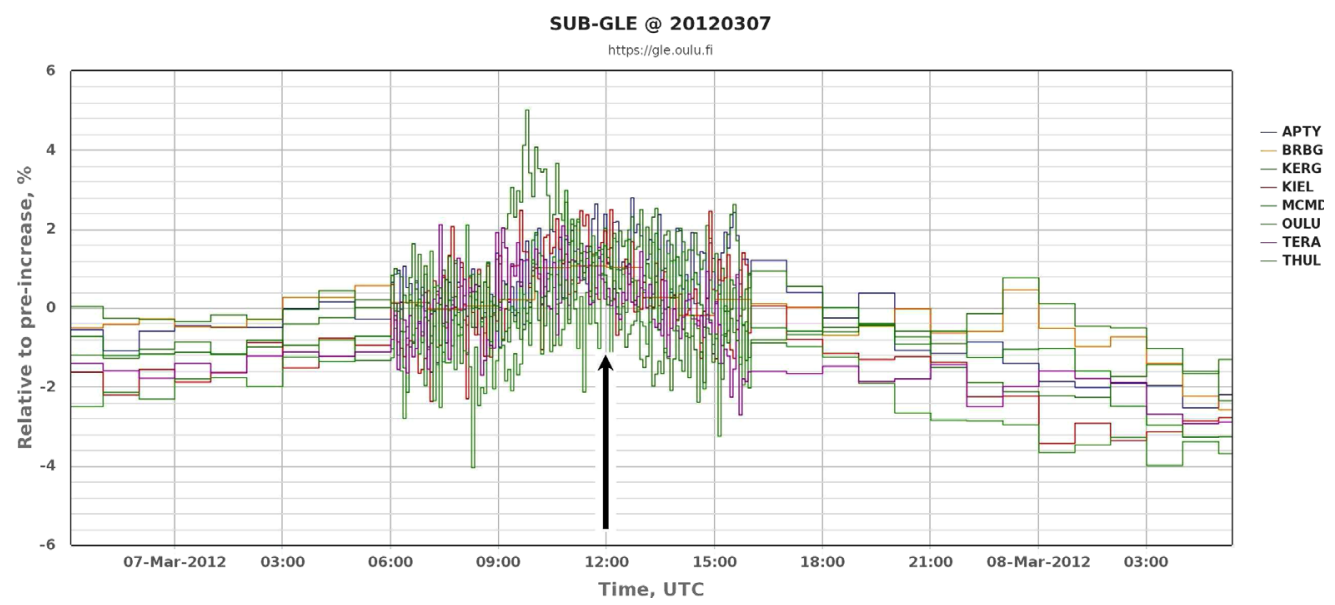

(b) NMs without statistically significant increase

Fig. A1. Profile of the time variation of NMs during sub-GLE event on 07 March 2012 as denoted in the legend. The arrow indicates the main phase of the event. (a) NMs with statistically significant increase compared with TERA. (b) NMs without statistically significant increase 


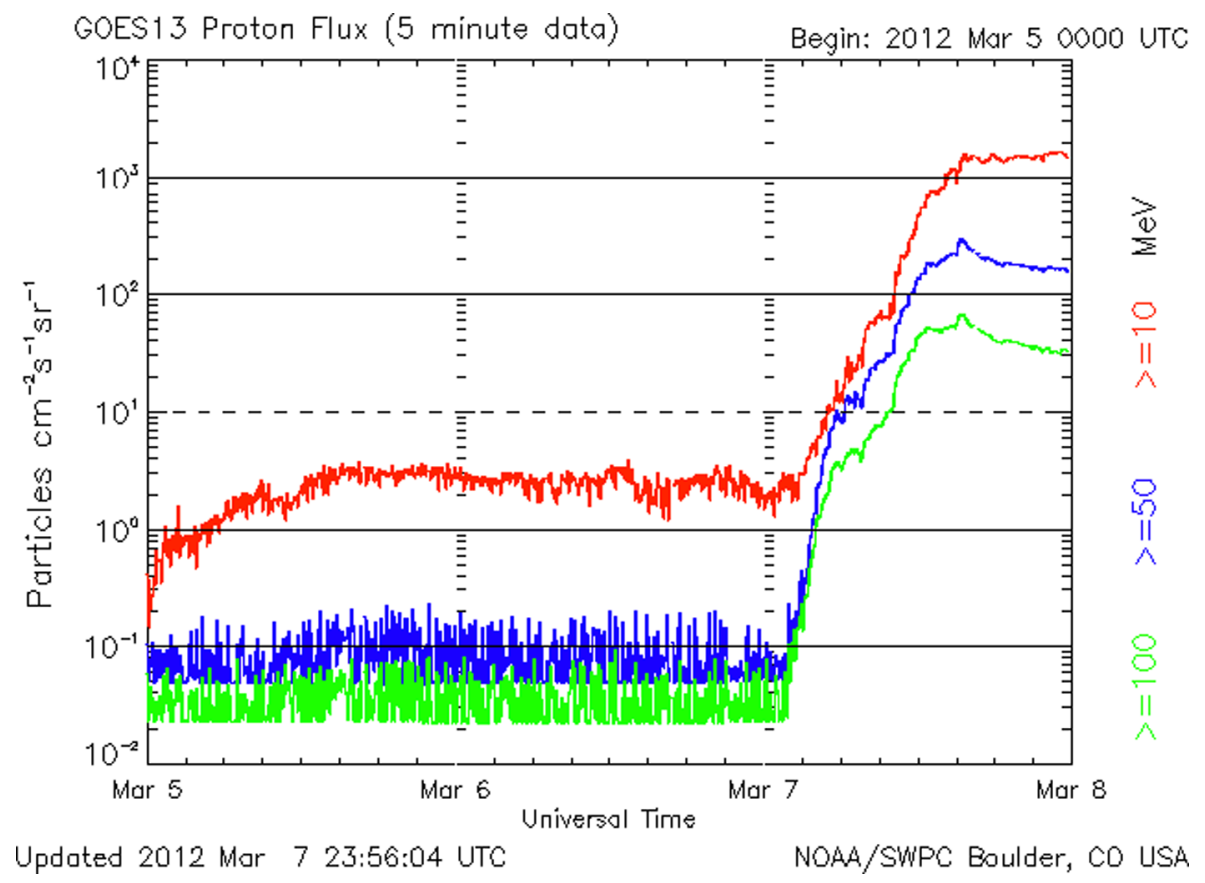

Fig. A2. Profile of the time variation of GOES 13 proton flux during sub-GLE event on 07 March 2012.

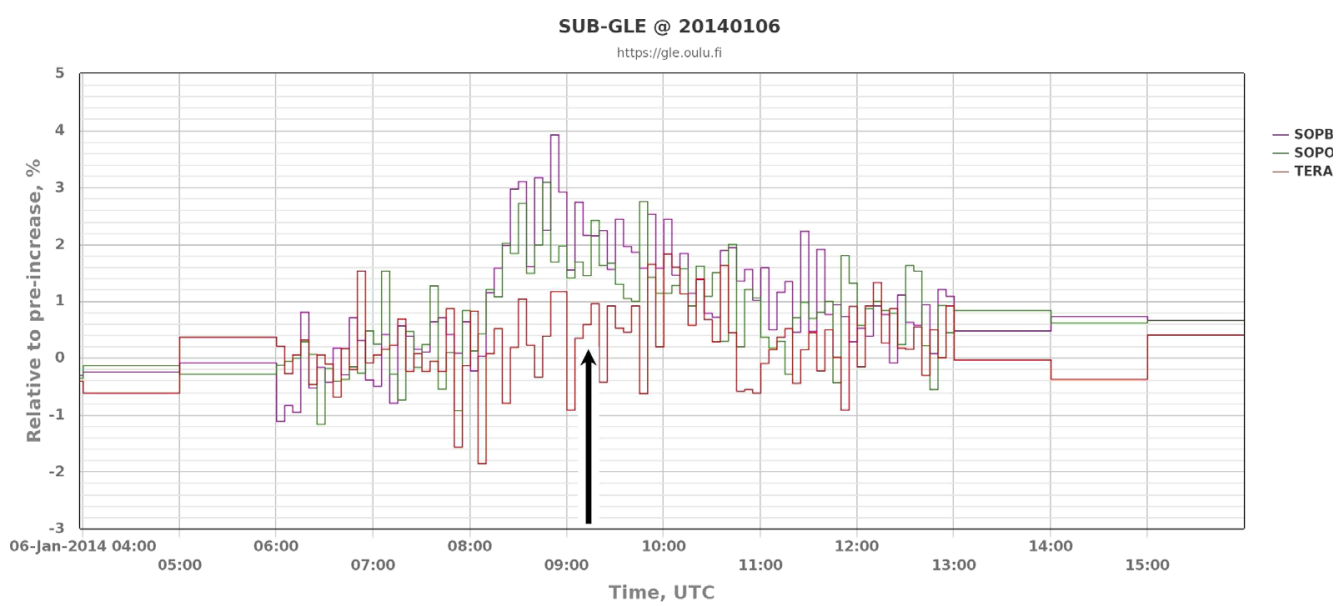

(a) NMs with statistically significant increase compared with TERA

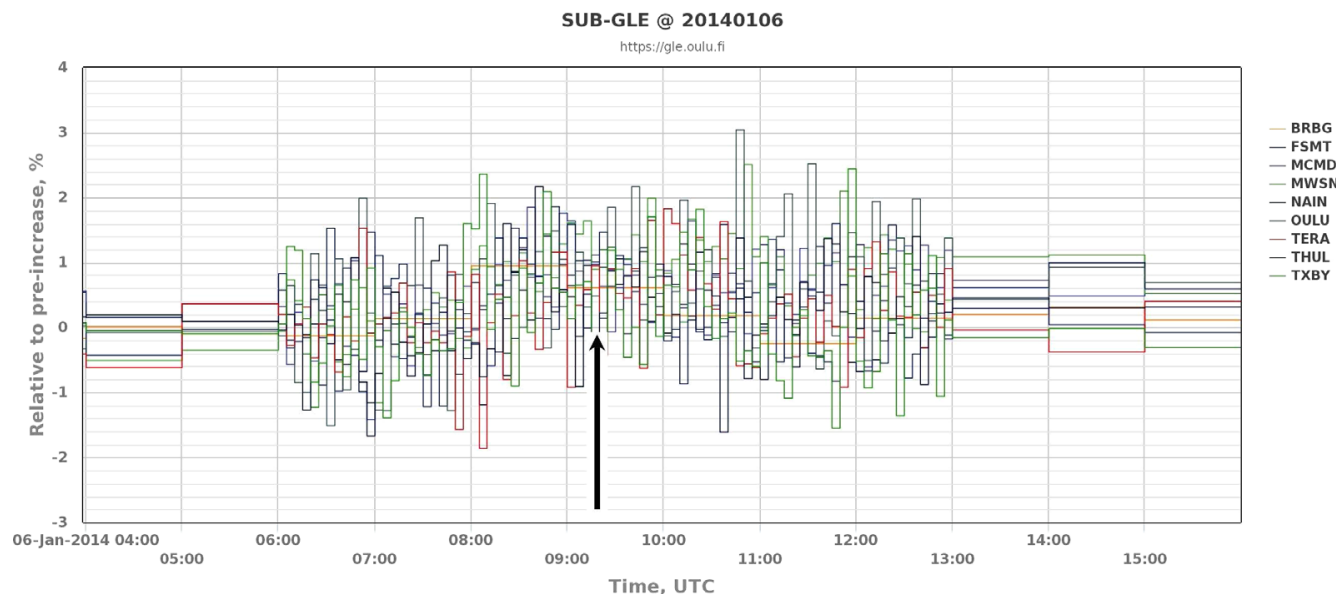

(b) NMs without statistically significant increase

Fig. A3. Profile of the time variation of NM during sub-GLE event on 06 January 2014 as denoted in the legend. The arrow indicates the main phase of the event. (a) NMs with statistically significant increase compared with TERA. (b) NMs without statistically significant increase. 
A. Mishev et al.: J. Space Weather Space Clim. 2017, 7, A28

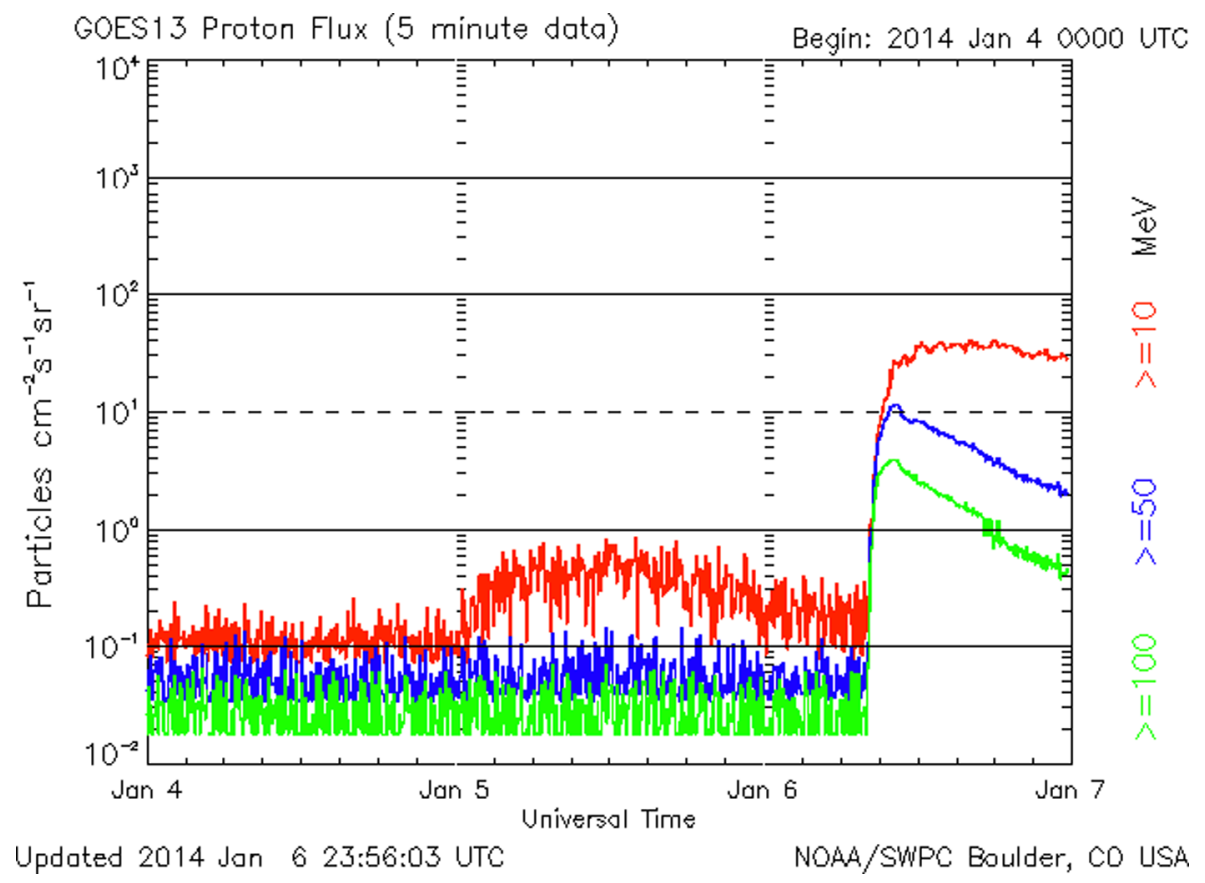

Fig. A4. Profile of the time variation of GOES 13 proton flux during sub-GLE event on 06 January 2014.

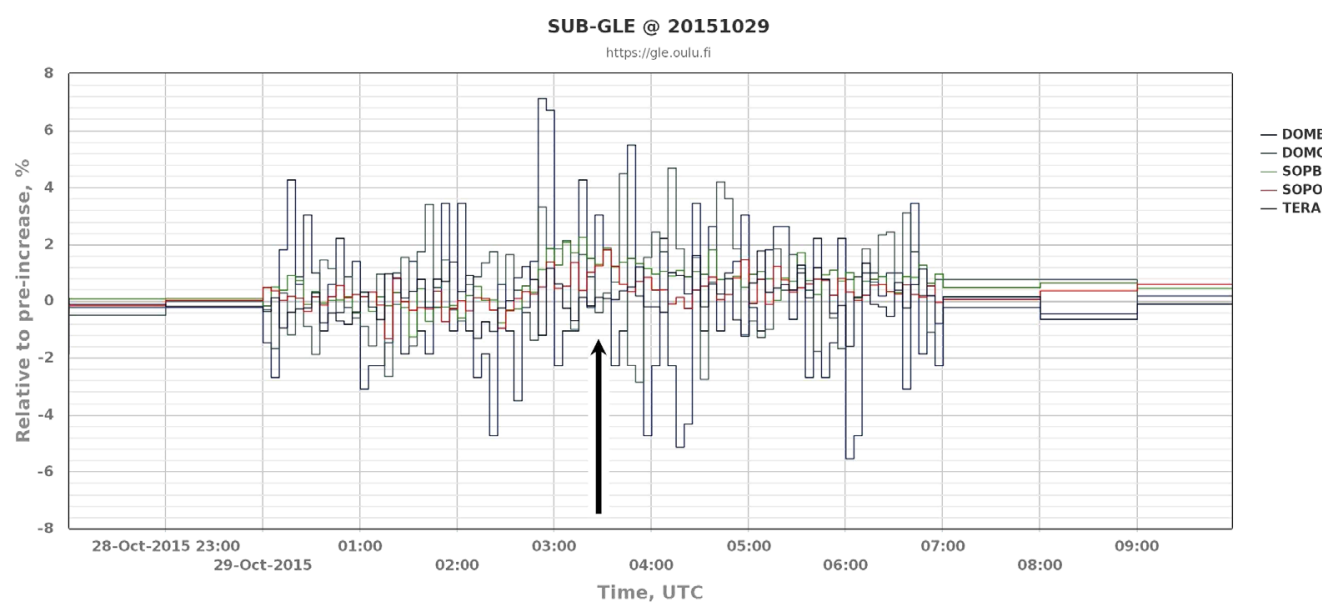

(a) NMs with statistically significant increase compared with TERA

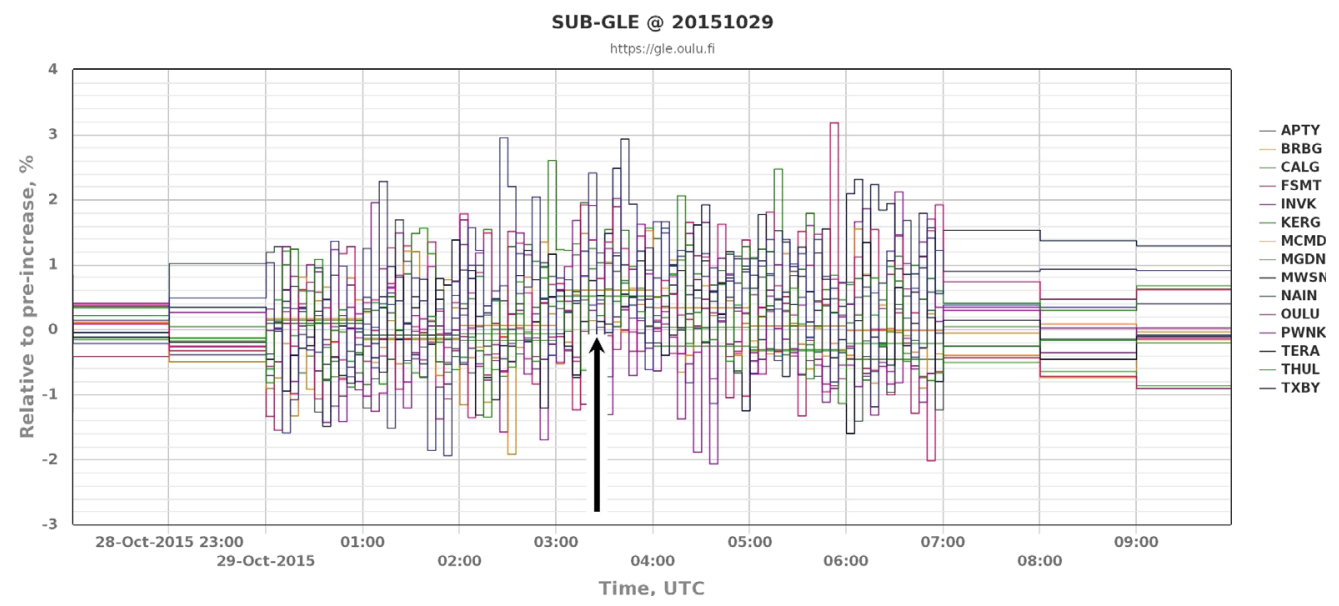

(b) NMs without statistically significant increase

Fig. A5. Profile of the time variation of NM during sub-GLE event on 29 October 2015 as denoted in the legend. The arrow indicates the main phase of the event. (a) NMs with statistically significant increase compared with TERA. (b) NMs without statistically significant increase. 


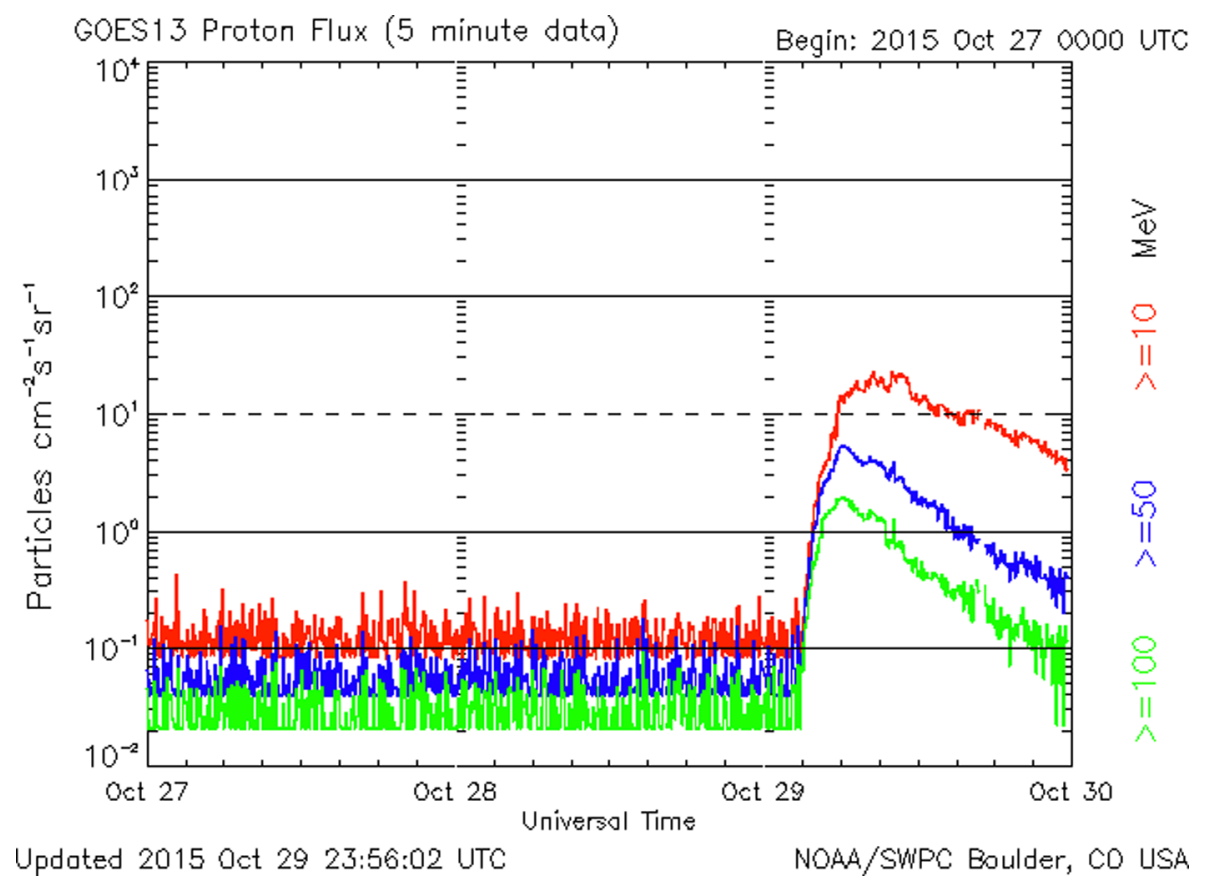

Fig. A6. Profile of the time variation of GOES 13 proton flux during sub-GLE event on 29 October 2015.

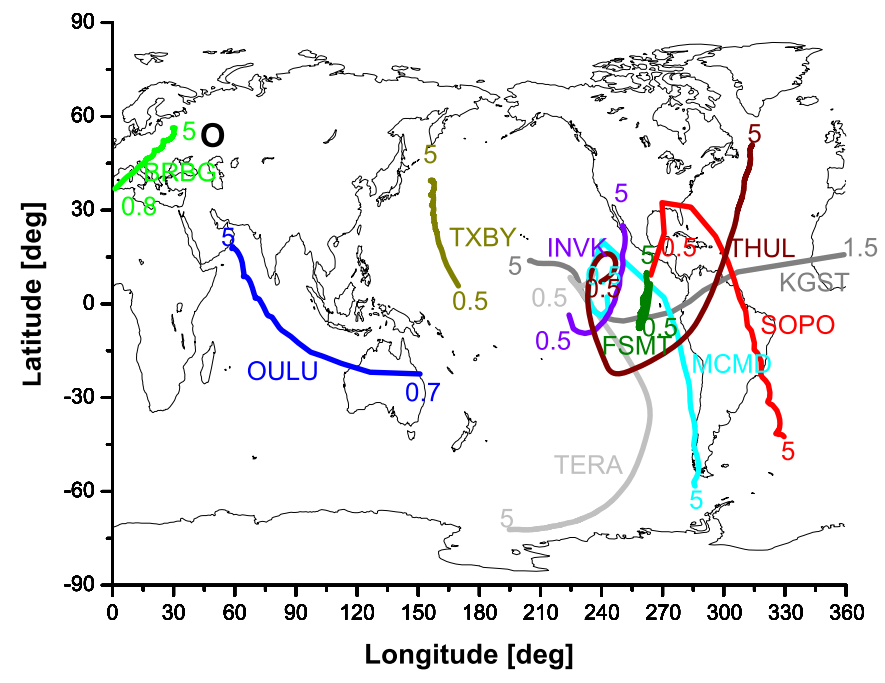

Fig. A7. Computed asymptotic directions for several NM stations during the sub-GLE event on 07 March 2012. The abbreviations (Tab. $\mathrm{B} 1$ ), the corresponding color lines and numbers indicate the NM stations and asymptotic directions, which are plotted in the rigidity range between the cut-off rigidity and $5 \mathrm{GV}$. The small oval corresponds to the direction of the IMF derived from the ACE satellite measurements during the event onset.

registered as sub-GLE, because the expected non-null response at Dome C NMs. The computed asymptotic directions used for the analysis of this event are shown in Figure A7, accordingly the contour plot of sum of variances for the best fit solutions vs. geographic latitude and longitude in Figure A9.

The event on 6 January 2014 is sometimes considered as a small GLE (Thakur et al., 2014; Li et al., 2016). However, according to our analysis, there is no statistically significant

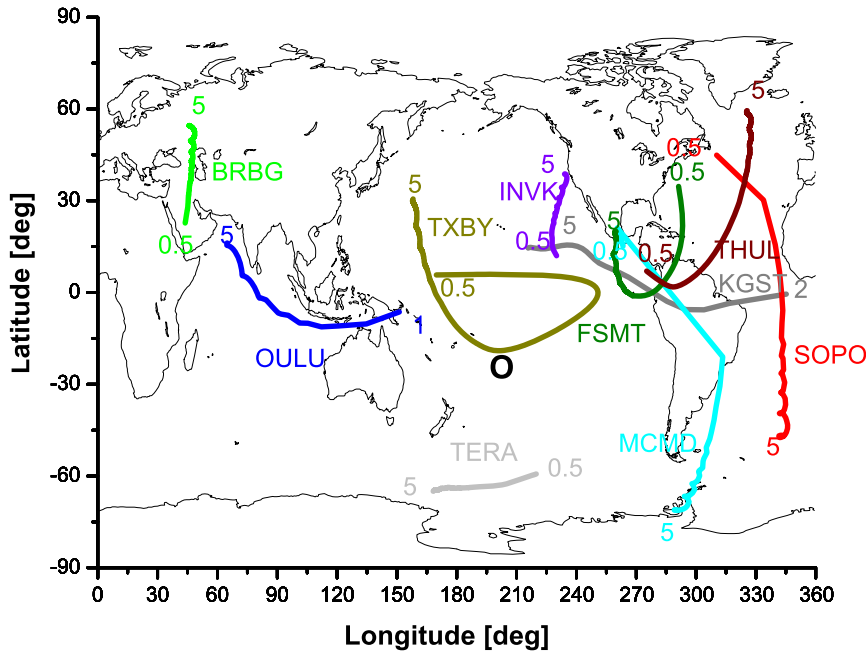

Fig. A8. Computed asymptotic directions for several NM stations during the sub-GLE event on 06 January 2014. The abbreviations (Tab. B1), the corresponding color lines and numbers indicate the NM stations and asymptotic directions, which are plotted in the rigidity range between the cut-off rigidity and $5 \mathrm{GV}$. The small oval corresponds to the direction of the IMF derived from the ACE satellite measurements during the event onset.

increase of count rates in any NMs, but South Pole (Fig. A3). Therefore, we classify this event as a candidate for a sub-GLE, similarly to the event on 7 March 2012. The event was well studied in the sense of satellite-borne signal, where an increase of proton flux was observed in all GOES channels (Fig. A4) (Li et al., 2016). The computed asymptotic directions used for the analysis of this event are shown in Figure A8, accordingly the contour plot of the sum of variances for the best fit solutions vs. 


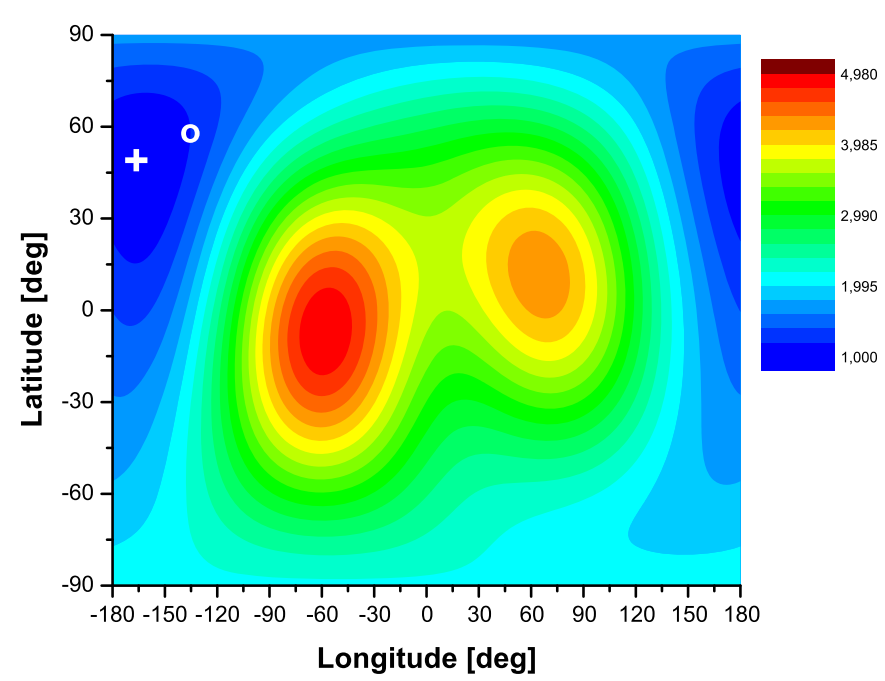

Fig. A9. Contour plot of $D$ (Eq. (6)) for the best fit solutions vs. geographic latitude and longitude during sub-GLE event on 07 March 2012 , normalized to the minimal value of $D$. The cross corresponds to the assumed apparent source position, while the small oval corresponds to the direction of the IMF derived from the ACE satellite measurements during the event onset.

geographic latitude and longitude in Figure A10. The assessed spectral and angular characteristics are presented in the main text (see Tab. 1 and Figs. 9 and 10).

Another event studied in this paper is the sub-GLE observed on 29 October 2015. The event was recorded with statistically significant increase only by the South Pole and

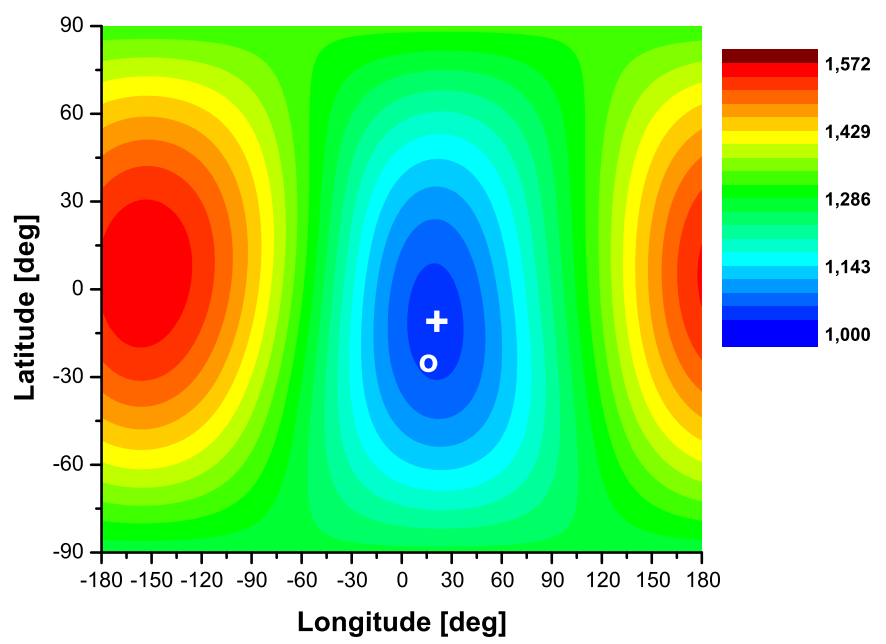

Fig. A10. Contour plot of $D$ (Eq. (6)) for the best fit solutions vs. geographic latitude and longitude sub-GLE event on 06 January2014, normalized to the minimal value of $D$. The cross corresponds to the assumed apparent source position, while the small oval corresponds to the direction of the IMF derived from the ACE satellite measurements during the event onset.

Dome C NMs (Fig. A5) and was accompanied by an increase of the proton flux in the GOES 13 data (Fig. A6). The analysis of the event is presented in the main text.

\section{Appendix B Neutron monitor stations used for the analysis of sub-GLE events}

Table B1. Neutron monitor station location with corresponding geomagnetic cut-off rigidity and altitude above sea level used in the analysis. The last three columns depict the dates of sub-GLE events. Cross (null) in the last three columns denote NMs used (not used) for the analysis of the event. The star denotes NMs with statistically significant increase.

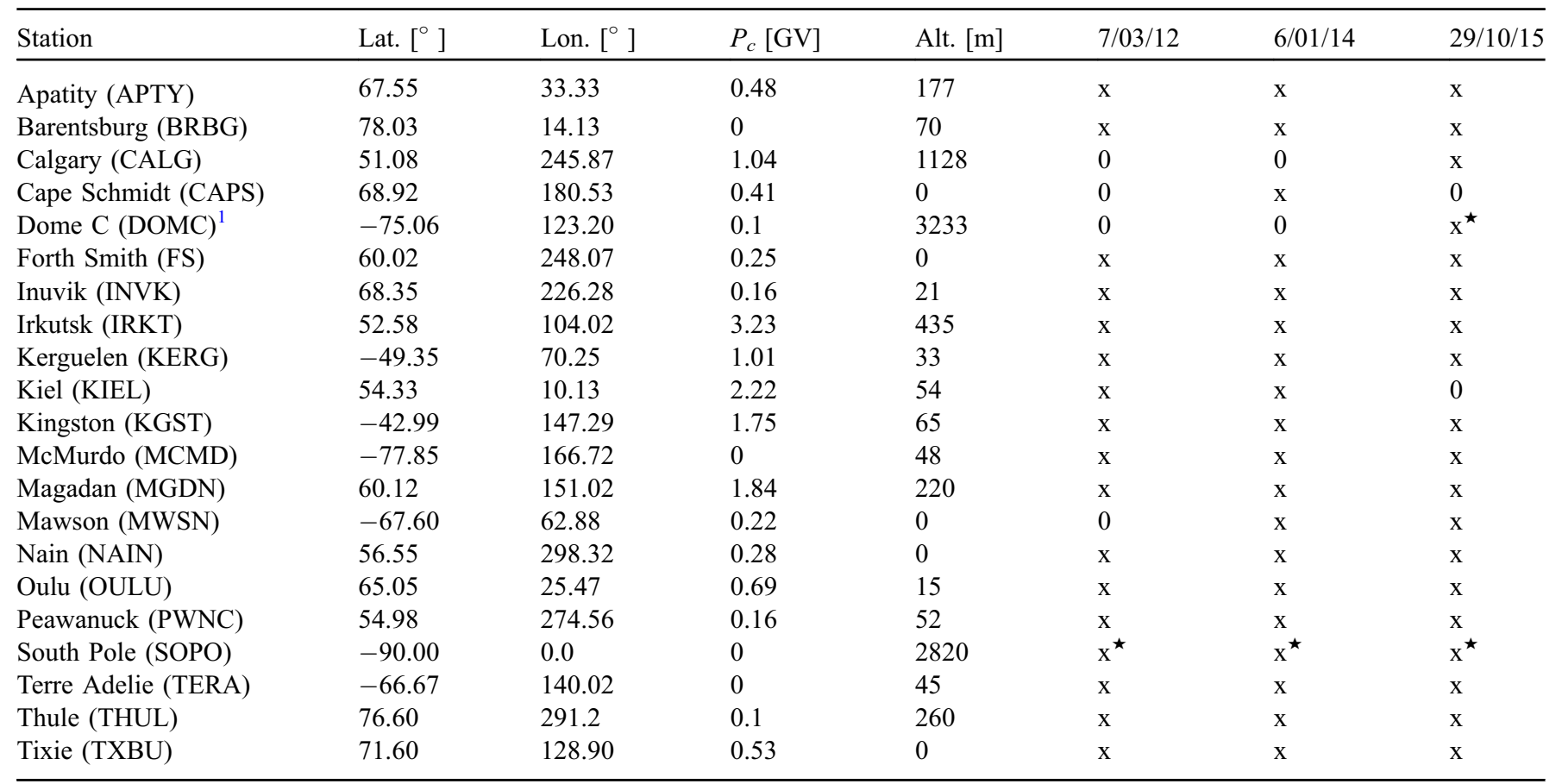

\footnotetext{
${ }^{1}$ The DOMC data are retrieved from the GLE database (gle.oulu.fi).
} 